\title{
Implementation of New Process Models for Tailored Polymer Composite Structures into Processing Software Packages
}

\author{
Ba Nghiep Nguyen ${ }^{(a)}$, Xiaoshi Jin ${ }^{(b)}$, Jin Wang ${ }^{(b)}$, Jay H Phelps ${ }^{(c)}$, \\ Charles L Tucker III ${ }^{(\mathrm{c})}$, Vlastimil Kunc ${ }^{(\mathrm{d})}$, Satish K Bapanapalli ${ }^{(\mathrm{a})}$, \\ Mark T Smith ${ }^{(a)}$
}
(a) Pacific Northwest National Laboratory, P.O. Box 999, Richland, WA 99352
(b) Autodesk, Inc. at Ithaca, NY 14850
(c) University of Illinois at Urbana-Champaign, Department of Mechanical Science and Engineering, Urbana, IL 61801
(d) Oak Ridge National Laboratory, P.O. Box 2009, Oak Ridge, TN 37831

February 2010

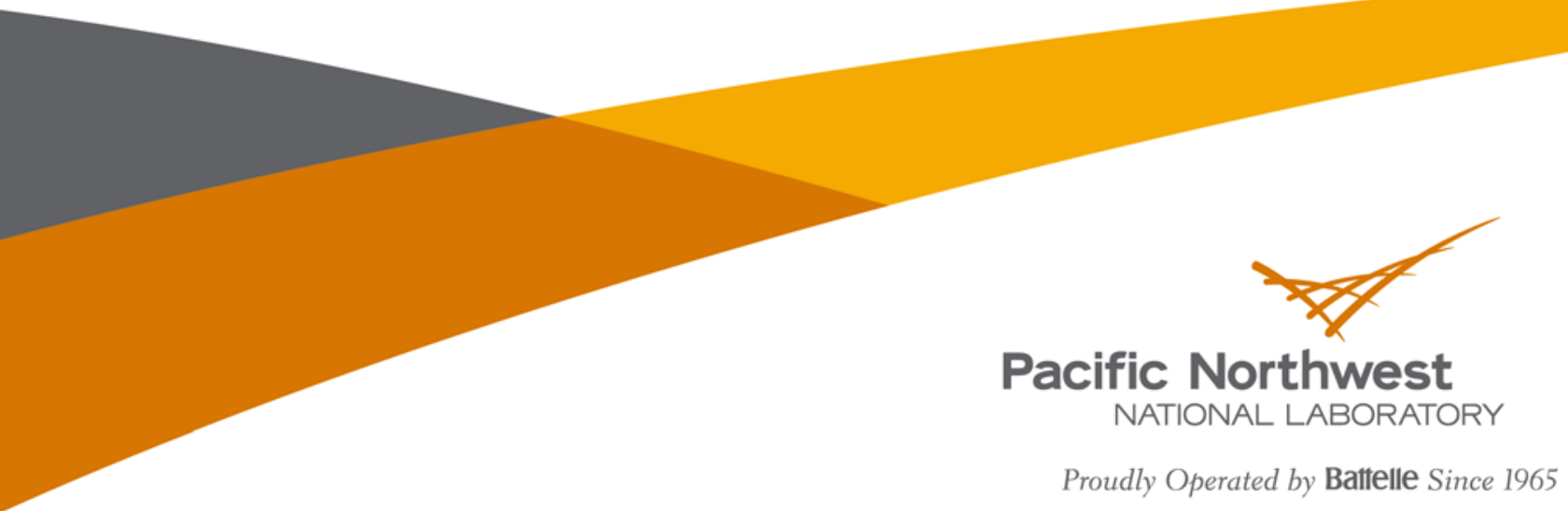




\section{DISCIAAIMER}

This report was prepared as an account of work sponsored by an agency of the United States Government. Neither the United States Government nor any agency thereof, nor Battelle Memorial Institute, nor any of their employees, makes any warranty, express or implied, or assumes any legal liability or responsibility for the accuracy, completeness, or usefulness of any information, apparatus, product, or process diselosed, or represents that its use would not infringe privately owned rights. Reference herein to any specific commercial product, process, or service by trade name, trademark, manufacturer, or otherwise does not necessarily constitute or imply its endorsement, recommendation, or favoring by the United States Government or any agency thereof, or Battelle Memorial Institute. The views and opinions of authors expressed herein do not necessarily state or rellect those of the United States Government or any agency thereof.

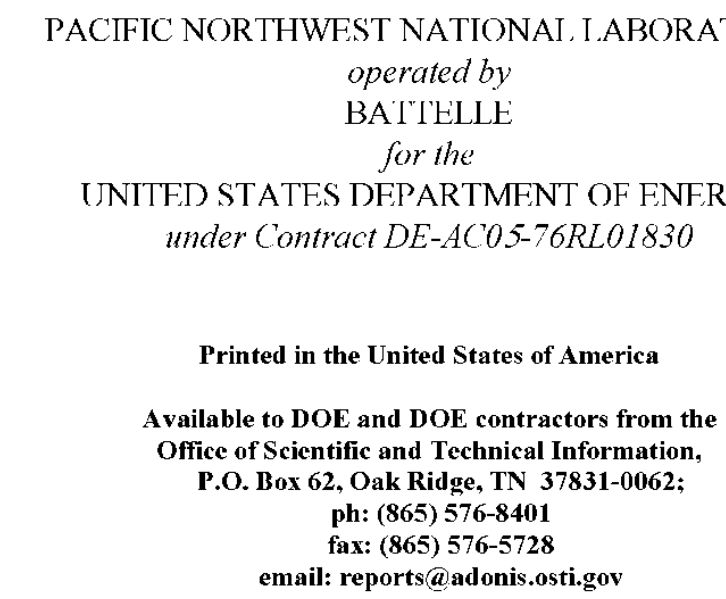

Available to the public from the National Technical Information Service, U.S. Department of Commerce, 5285 Port Royal Rd., Springfield, VA 22161 ph: (800) 553-6847 fax: (703) 605-6900 email: orders@ntis.fedworld.gov online ordering: http://www.ntis.gov/ordering.htm

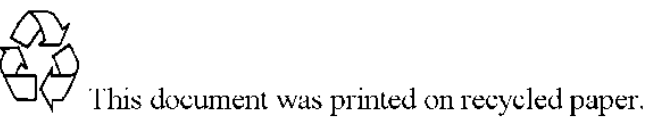

(9/2003) 


\title{
Implementation of New Process Models for Tailored Polymer Composite Structures into Processing Software Packages
}

\author{
Ba Nghiep Nguyen $^{(a)}$, Xiaoshi Jin ${ }^{(b)}$, Jin Wang ${ }^{(b)}$, Jay H Phelps ${ }^{(c)}$, \\ Charles L Tucker III ${ }^{(\mathrm{c})}$, Vlastimil Kunc ${ }^{(\mathrm{d})}$, Satish K Bapanapalli( ${ }^{(\mathrm{a})}$, \\ Mark T Smith ${ }^{(a)}$
}

\footnotetext{
(e) Pacific Northwest National Laboratory, P.O. Box 999, Richland, WA 99352

(f) Autodesk, Inc. at Ithaca, NY 14850

(g) University of Illinois at Urbana-Champaign, Department of Mechanical Science and Engineering, Urbana, IL 61801

(h) Oak Ridge National Laboratory, P.O. Box 2009, Oak Ridge, TN 37831
}

February 2010

Prepared for

the U.S. Department of Energy

under Contract DE-AC05-76RL01830 



\section{Executive Summary}

Recently, injection molded long-fiber thermoplastics (LFTs) have generated great interest within the automotive industry as these materials can potentially be used for structural applications to reduce vehicle weight. However, before our work on LFTs, injection-molding of these materials posed a great challenge because of two main reasons: (i) no process models for LFTs had been developed that could predict the injection-molding of an LFT part, and (ii) no experimental characterization methods had been developed to enable the characterization of the as-formed LFT microstructure to determine the fiber orientation and length distributions that are critical to any process model development. The objective of this project is two-fold. First, the advanced process models for LFTs are developed that can accurately predict the composite microstructure governed by the flow-induced fiber orientation, fiber length distribution, and other features resulting from processing. Second, the models are implemented in the Autodesk Moldflow Insight software to enable the injection molding simulations of LFT structures.

This report describes the work conducted under the Cooperative Research and Development Agreement (CRADA) (Nr. 260) between Battelle as Operator of the Pacific Northwest National Laboratory (PNNL) and Autodesk, Inc. PNNL has worked with the University of Illinois at Urbana-Champaign (UIUC) through subcontracts to develop process models for fiber orientation and length distributions in injection-molded LFTs. The UIUC has then developed and implemented the new process models for LFTs in an in-house code named ORIENT to enable first validations and applications of these models. Next, Autodesk, Inc. has implemented these new process models in Autodesk Moldflow Insight for LFT injection molding simulations. In addition, Autodesk, Inc. has performed rheological and mechanical tests to identify the rheological and physical properties for the pellet materials used in this CRADA. These properties are needed for injection-molding simulations using Autodesk Moldflow Insight. Autodesk, Inc. has delivered to PNNL the Autodesk Moldflow Insight research versions containing the newly implemented models for process simulations and also has provided PNNL with the technical support for the model validations. UIUC has assisted Autodesk, Inc. in the model implementations in Autodesk Moldflow Insight and also has assisted PNNL in the model validation using Autodesk Moldflow Insight by providing consultancy and reviews. Oak Ridge National Laboratory (ORNL) has performed microstructural characterizations for fiber length and orientation allowing the validations of the developed models. 


\section{Contents}

Executive Summary...............................................................................................................iii

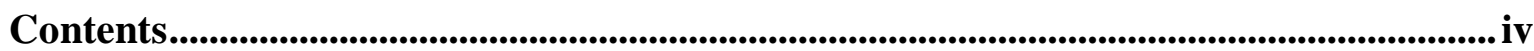

List of Figures.......................................................................................................................

List of Tables ..........................................................................................................................

1.0 Introduction ................................................................................................................................ 1

2.0 Fiber Orientation Models Developed for SFTs .................................................................

2.1 The Folgar-Tucker Model .....................................................................................

2.2 The Wang et al. Model ........................................................................................

3.0 Assessment of Fiber Orientation Models for SFTs ......................................................2

3.1 Rheological and Physical Properties ........................................................................ 4

3.2 Correlations between Model Predictions and Experimental Results ........................6

4.0 The Anisotropic Rotary Diffusion - Reduced Strain Closure Model........................10

5.0 Development of A Fiber Length Attrition Model in the Mold ...................................13

6.0 Implementations of New Process Models in Autodesk Moldflow Insight ..............15

6.1 Fiber Orientation Distribution Model Implementations...........................................16

6.2 Fiber Length Attrition Model Implementation ………………………………….....29

7.0 Dialogue Box Designs for FOD and FLD ......................................................................33

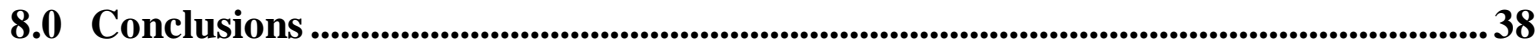

9.0 Acknowledgements ........................................................................................................ 39

10.0 References ..................................................................................................................................... 39 


\section{List of Figures}

Figure 1 The 3mm thick injection-molded glass/PP center-gated disk (a) and ISO-plaque (b): Regions A, B, and C being $25.4 \mathrm{~mm}$ long along the flow directions were taken for fiber orientation and length measurements.....

Figure 2 Viscosity vs. strain rate for the studied glass-fiber/PP at four temperatures [12]............ 4

Figure $3 A_{11}$ vs. non-dimensional thickness coordinate $z / b$ for the AF3I ISO-plaque (Table 1 ). The experimental data is compared against three different sets of parameters for orientation modeling. The best fit is provided by the RSC model with a SRF factor much greater than one and $C_{\mathrm{I}}=0.03$.

Figure $4 A_{11}$ vs. non-dimensional thickness coordinate $\mathrm{z} / \mathrm{b}$ for $\mathrm{AF3I}$ at Region $\mathrm{B}$. $S R F=30$ and $C_{\mathrm{I}}=0.03$.

Figure $5 A_{11}$ vs. non-dimensional thickness coordinate $\mathrm{z} / \mathrm{b}$ for AS3I at Region B. $S R F=30$ and $C_{\mathrm{I}}=0.03$.

Figure $6 A_{11}$ vs. non-dimensional thickness coordinate $z / b$ for AF3D at Region B. $S R F=30$ and $C_{\mathrm{I}}=0.03$

Figure $7 A_{11}$ vs. non-dimensional thickness coordinate $z / b$ for AS3D at Region B. $S R F=30$ and $C_{\mathrm{I}}=0.03$.

Figure $8 A_{22}$ and $A_{33}$ vs. non-dimensional thickness coordinate $z / b$ for AS3I at Region B. $S R F=$ 30 and $C_{I}=0.03$.

Figure 9 Predicted (by the ARD-RSC model implemented in ORIENT) and measured fiber orientation tensor components $A_{11}$ and $A_{22}$ for Region B of the AF3I ISO-plaque.

Figure 10 Predicted (by the ARD-RSC model implemented in ORIENT) and measured fiber orientation tensor components $A_{33}$ and $A_{31}$ for Region B of the AF3I ISO-plaque.

Figure 11 Measured number of fibers vs. fiber length for a glass-fiber/PP disk (AF3D), compared to predictions of the fiber length model.

Figure 12 Predicted and experimental average fiber lengths for the same glass-fiber/PP disk shown in Figure 11.

Figure 13. The default inlet orientation to match the long-fiber orientation data......

Figure 14 Fiber orientations predicted by the ARD-RSC model with $\kappa=0.05$ for an end-gated plaque modeled with different mid-plane mesh sizes. The results are obtained from the previous special build prior to the gradient modification of Autodesk Moldflow Insight. .. 17

Figure 15 Fiber orientations predicted by the ARD-RSC model with $\kappa=0.05$ for an end-gated plaque modeled with different mid-plane mesh sizes. The results are obtained from the special build with the modification of the gradient calculations.

Figure 16 A three-mm thick end-gated plaque (AF3I or AS3I) modeled by a mid-plane mesh.... 19

Figure 17 A three-mm thick center-gated disk (AF3D or AS3D) modeled by a mid-plane mesh. 19

Figure 18 A three-mm thick end-gated plaque (AF3I or AS3I) modeled by a 3D mesh.............. 20

Figure 19 A three-mm thick center-gated disk (AF3D or AS3D) modeled by a 3D mesh........... 20

Figure 20 Comparison of fiber orientation data with the prediction by the ARD-RSC model with $\kappa=1 / 30$ for the AF3I ISO-plaque (fast fill) using a mid-plane mesh.................................. 21 
Figure 21 Comparison of fiber orientation data with the prediction by the ARD-RSC model with $\kappa=1 / 30$ for the AS3I ISO-plaque (slow fill) using a mid-plane mesh.

Figure 22 Comparison of fiber orientation data with the prediction by the ARD-RSC model with $\kappa=1 / 30$ for the AF3D disk (fast fill) using a mid-plane mesh.

Figure 23 Comparison of fiber orientation data with the prediction by the ARD-RSC model with $\kappa=1 / 30$ for the AS3D disk (slow fill) using a mid-plane mesh. 24

Figure 24 Comparison of fiber orientation data with the prediction by the ARD-RSC model with $\kappa=1 / 30$ for the AF3I plaque (fast fill) using a 3D mesh.

Figure 25 Comparison of fiber orientation data with the prediction by the ARD-RSC model with $\kappa=1 / 30$ for the AS3I plaque (slow fill) using a 3D mesh.

Figure 26 Comparison of fiber orientation data with the prediction by the ARD-RSC model with $\kappa=1 / 30$ for the AF3D disk (fast fill) using a 3D mesh.

Figure 27 Comparison of fiber orientation data with the prediction by the ARD-RSC model with $\kappa=1 / 30$ for the AS3D disk (slow fill) using a 3D mesh..................................................... 28

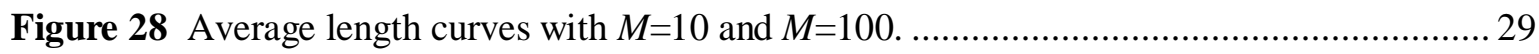

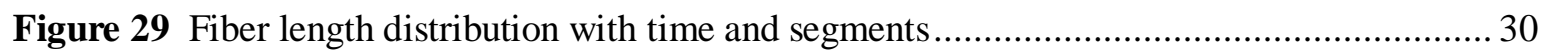

Figure 30 Fiber breakage results in two cross sectional views................................................ 32

Figure 31 Fiber breakage result at the end of cavity............................................................... 32

Figure 32 Fiber breakage results in a rib and at the top of the rib......................................... 32

Figure 33 Dialog box for fiber length information data.................................................... 333

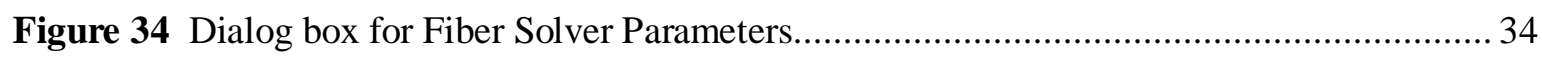

Figure 35 Fiber length attrition model parameters in Fiber Solver Parameters dialog box. .......... 35

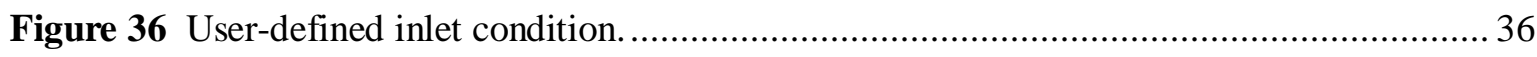

Figure 37 Dialog box for the RSC and ARD models' parameters. .......................................... 37

Figure 38 “New Plot” selection for for fiber length results from "Results”. ............................. 37

Figure 39 Fiber length distribution result selected from the complete result list. ....................... 38

\section{List of Tables}

Table 1 Summary of materials, injection speed, and mold geometry for each of the molded samples.....4 Table 2 Specific heat $\mathrm{c}_{\mathrm{p}}$ over a range of temperatures $\mathrm{T}$ for the glass-fiber/PP material as reported by

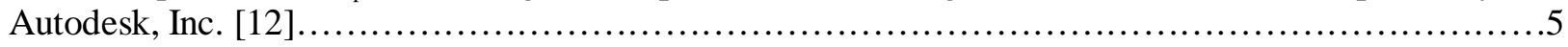

Table 3 Thermal conductivity k over a range of temperatures $\mathrm{T}$ for the glass-fiber/PP material as reported

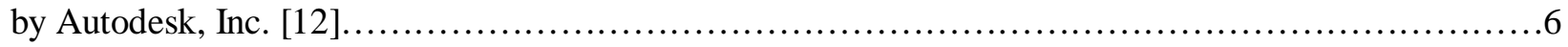

Table 4 Fitting parameters for the Cross-WLF model for the studied glass-fiber/PP material as reported

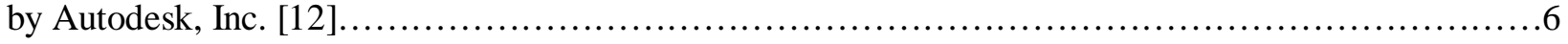




\subsection{Introduction}

The structure of this report is organized as follows. After the Introduction Section (Section 1), Section 2 summarizes the current fiber orientation models developed for injection-molded short-fiber thermoplastics (SFTs) [1-4]. Section 3 provides an assessment of these models to determine their capabilities and limitations, and the developments needed for injection-molded LFTs. Section 4 then focuses on the development of a new fiber orientation model for LFTs. This model is termed the anisotropic rotary diffusion - reduced strain closure (ARD-RSC) model [5-6] as it explores the concept of anisotropic rotary diffusion to capture the fiber-fiber interaction in long-fiber suspensions and uses the reduced strain closure method of Wang et al. [3] to slow down the orientation kinetics in concentrated fiber suspensions. In contrast to fiber orientation modeling, before this project, no standard model was developed to predict the fiber length distribution in molded fiber composites. Section 5 is therefore devoted to the development of a fiber length attrition model in the mold [5-6]. Sections 6 and 7 address the implementations of the models in Autodesk Moldflow Insight, and the conclusions drawn from this work is presented in Section 8.

\subsection{Fiber Orientation Models Developed for SFTs}

\subsection{The Folgar-Tucker Model}

For SFTs, Folgar and Tucker [1] added an isotropic rotary diffusion term to Jeffrey's equation (for dilute concentration) to represent the randomizing effect of the fiber-fiber interaction in concentrated suspensions. This term depicts the hydrodynamic fiber-fiber interaction. Later, Advani and Tucker [2] recast the Folgar-Tucker equation in terms of the fiber orientation tensor components as:

$$
\frac{D A_{i j}}{D t}+\frac{1}{2}\left(\omega_{i k} A_{k j}-A_{i k} \omega_{k j}\right)=\frac{1}{2} \kappa\left(\dot{\gamma}_{i k} A_{k j}+A_{i k} \dot{\gamma}_{k j}-2 \dot{\gamma}_{k l} A_{i j k l}\right)+2 C_{1} \dot{\gamma}\left(\delta_{i j}-3 A_{i j}\right)
$$

where $A_{i j}$ and $A_{i j k l}$ are the second and fourth-order orientation tensors, respectively. $\omega_{i j}$ is the vorticity tensor, and $\dot{\gamma}_{i j}$ is the rate of the deformation tensor whose scalar magnitude is $\dot{\gamma} . \kappa$ and $C_{\mathrm{I}}$ are material constants; $\kappa$ depends on the fiber aspect ratio $r$, and $C_{\mathrm{I}}$ is called the interaction coefficient. If $C_{I}=0$ and $\kappa=\left(r^{2}-1\right) /\left(r^{2}+1\right)$, Equation (1) is then Jeffrey's equation for the motion of a rigid ellipsoidal shape fiber in a Newtonian solvent. This is strictly valid for dilute suspensions in which the fiber-fiber interaction is absent or negligible. $C_{\mathrm{I}}$ can be identified by fitting the predicted orientation results for the component $A_{11}$ to the corresponding experimental data. A closure approximation [8-9] is used to estimate the fourth-order tensor $A_{i j k l}$ from $A_{i j}$. Bay [9] has identified $C_{\mathrm{I}}$ for a set of short-glass fiber thermoplastics and has found that $C_{\mathrm{I}}$ initially increases as the fiber volume fraction or aspect ratio increases but beyond a certain value of $\mathrm{cr}$ ( $c r>1$, where $c$ is the concentration), $C_{\mathrm{I}}$ decreases with increasing concentration. This can be 
explained by considering the screening effect that attenuates the disturbance at higher concentrations. The disturbance induced by the presence of a fiber is screened by the other fibers resulting in decreasing the fiber-fiber interaction, hence decreasing $C_{\mathrm{I}}$.

\subsection{The Wang et al. Model}

Wang et al. [3] developed a procedure termed as "reduced strain closure" method to slow down the orientation kinetics in concentrated short-fiber suspensions. According to Wang et al. [3], the rate of the second-order orientation tensor reads:

$$
\begin{aligned}
& \frac{D A_{i j}}{D t}=\frac{1}{2}\left(A_{i k} \omega_{k j}-\omega_{i k} A_{k j}\right)+\frac{\kappa}{2}\left(A_{i k} \dot{\gamma}_{k j}+\dot{\gamma}_{i k} A_{k j}-2\left[A_{i j k l}+\left(1-\frac{1}{S R F}\right)\left(L_{i j k l}-M_{i j m n} A_{m n k l}\right)\right] \dot{\gamma}_{k l}\right) \\
& +\frac{2 C_{I} \dot{\gamma}}{S R F}\left(\delta_{i j}-3 A_{i j}\right)
\end{aligned}
$$

$\kappa$ depends on the fiber aspect ratio and for long rigid cylinders is approximately equal to $\kappa=\left(r^{2}-1\right) /\left(r^{2}+1\right)$. In case of slender particles (e.g. long fibers), $\kappa$ approaches unity, and the presence of the interaction term inhibits fiber tumbling; hence the fiber length does not affect fiber orientation through this parameter, and $\kappa=1$ is used. $S R F$ is an empirical parameter termed the strain reduction factor that is introduced to reduce the rate of fiber alignment in concentrated fiber suspensions. If $S R F=1$, the Folgar-Tucker [1-2] model is recovered. In practice, coefficients $C_{\mathrm{I}}$ and $S R F$ are identified by fitting the predicted result for $A_{11}$ to the measured value for this component. $L_{i j k l}$ and $M_{i j k l}$ are fourth-order tensors formed from the eigenvalues and eigenvectors of $A_{i j}$ [3]. In this report, Wang et al.'s model is termed the reduced strain closure (RSC) model.

\subsection{Assessment of Fiber Orientation Models for SFTs}

This section applies the above models developed for SFTs to predict fiber orientation for a long-glass-fiber/polypropylene material, using two different mold geometries: an end-gated strip and a center-gated disk [4]. In order to compute the orientation state for an injection molding operation, the equations of balance of mass, momentum, and energy must be solved so that a velocity field can be computed. A program named ORIENT developed by the UIUC was used to solve for the velocity profile and the orientation in the above-mentioned geometries. A detailed description of the theory and numerical methods behind this program is found in [10]. ORIENT uses the Hele-Shaw approximation for solving for the velocity field in mold-filling operations where the velocity solution is decoupled from the orientation solution. The Hele-Shaw approximations are used to reduce the fully three-dimensional (3-D) flow problem to a twodimensional one for the pressure. These approximations are good for flows in thin cavities.

Table 1 provides a concise summary of the molded samples studied in this Section. They are the 3-mm thick center-gated disks and 3-mm thick ISO-plaques injection-molded under slow fill

and fast fill conditions. The material used for injection-molding has a polypropylene (PP) matrix 
with a $40 \%$ weight fraction of glass fibers. The center-gated disk is $177.8 \mathrm{~mm}$ in diameter, while the ISO plaque is $90 \mathrm{~mm}$ long and $80 \mathrm{~mm}$ wide. In each of the samples, the mold temperature was held to approximately $70{ }^{\circ} \mathrm{C}$ while the inlet temperature of the melt was $238^{\circ} \mathrm{C}$.

Figures 1(a) and 1(b) present the injection-molded disk and plaque samples. In order to compare the predicted and measured orientations, each of the samples presented in Table 1 were analyzed at three 25.4-mm-long regions denoted A, B, and C. For the ISO plaques, Region A was centered near the inlet at $x=15 \mathrm{~mm}$, Region B was centered approximately half-way down the length of the plaque at $x=45 \mathrm{~mm}$, and region $\mathrm{C}$ was closer to the end of the plaque, centered at $x=75 \mathrm{~mm}$. Each of the regions was located centrally in the cross-flow direction. For the center-gated disk, Region A was located near the inlet at $R=6 \mathrm{~mm}$, Region B was at $R=34 \mathrm{~mm}$, and Region $\mathrm{C}$ was closer to the edge of the disk at $R=64 \mathrm{~mm}$, where $R$ is the radius from the center of the disk. The measured orientation data was computed at 21 slices across the thickness of the part.

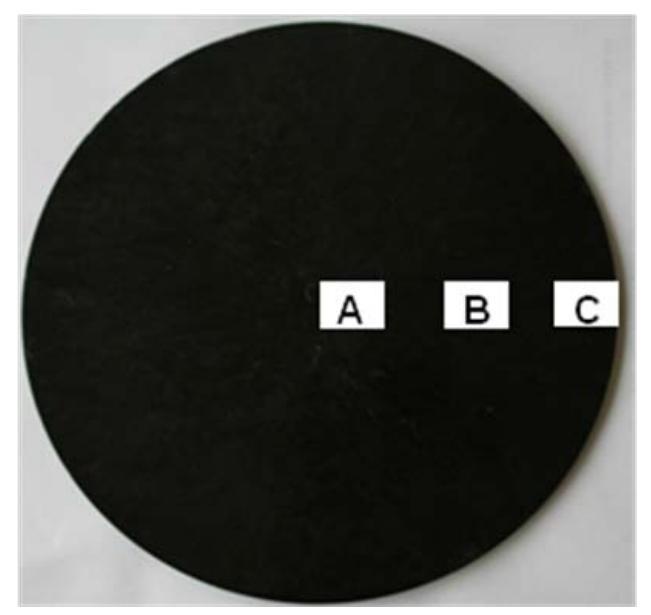

(a)

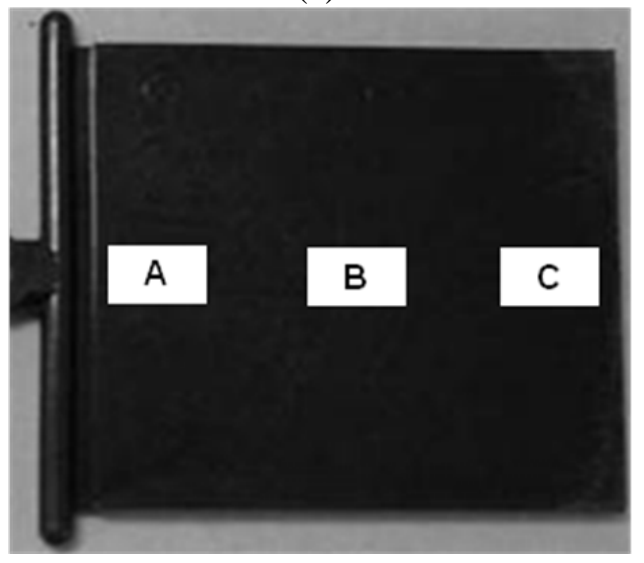

(b)

Figure 1 The 3mm thick injection-molded glass/PP center-gated disk (a) and ISO-plaque (b): Regions A, B, and C being $25.4 \mathrm{~mm}$ long along the flow directions were taken for fiber orientation and length measurements. 


\subsection{Rheological and Physical Properties}

Autodesk, Inc in Ithaca, New York performed the rheological and mechanical testing and supplied the appropriate rheological and thermal properties for the study. The density of glass/PP material was reported as $1.2203 \mathrm{~g} / \mathrm{cm}^{3}$. Tables 2 and 3 give the specific heats $c_{\mathrm{p}}$ and thermal conductivities $k$, respectively, for this material over a range of temperatures. The material viscosity was reported to obey a Cross-WLF relationship with respect to strain rate and temperature, and the appropriate parameters were also calculated and reported by Autodesk, Inc. (formerly Moldflow, Inc.) [12].

Table 1 Summary of materials, injection speed, and mold geometry for each of the molded samples.

\begin{tabular}{ccccc}
\hline \hline \multirow{2}{*}{$\begin{array}{c}\text { Sample } \\
\text { Code }\end{array}$} & Material & Fill Speed & Fill Time [s] & Geometry \\
\hline AF3D & Glass-fiber/PP & Fast & 0.65 & Disk \\
AS3D & Glass-fiber/PP & Slow & $4.79-4.18$ & Disk \\
AF3I & Glass-fiber/PP & Fast & 0.48 & ISO Plaque \\
AS3I & Glass-fiber/PP & Slow & 3.33 & ISO Plaque
\end{tabular}

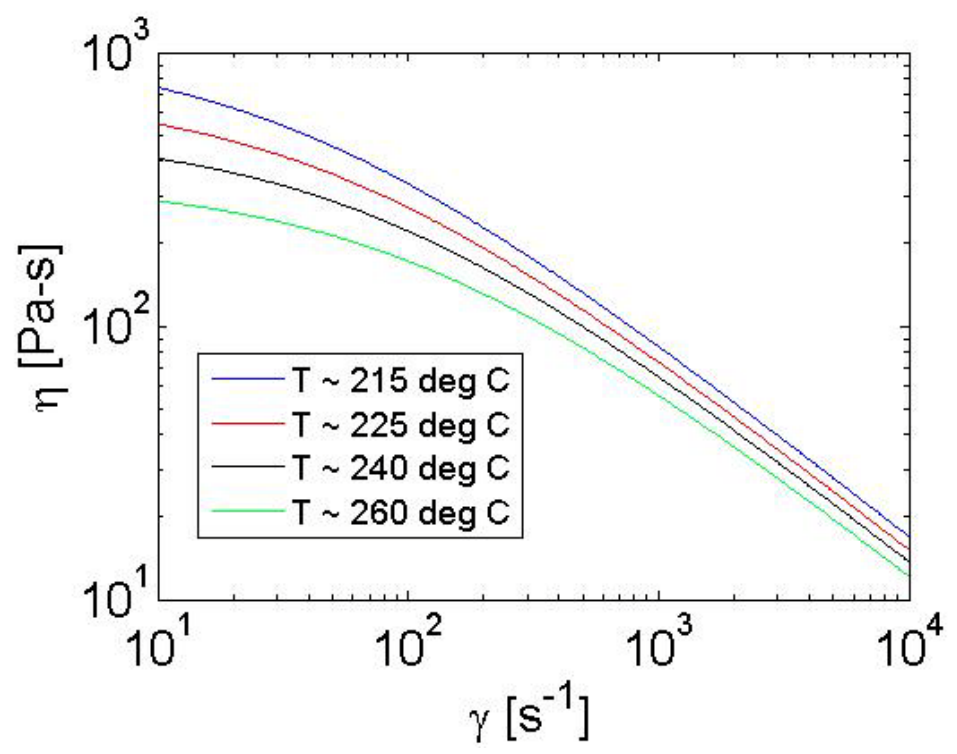

Figure 2 Viscosity vs. strain rate for the studied glass-fiber/PP at four temperatures [12].

With this relationship, the viscosity $\eta$ is related to the strain rate $\dot{\gamma}$ (i.e., the scalar magnitude of the rate-of-deformation tensor) through the Cross-WLF model which is 


$$
\eta=\eta_{0}\left[1+\left(\frac{\eta_{0} \dot{\gamma}}{\tau *}\right)^{1-n}\right]
$$

where $\tau^{*}$ and $n$ are the fitting parameters. This is a special case of the Cross-Carreau model. The WLF equation relates the reference viscosity $\eta_{0}$ to temperature $T$ and is given as

$$
\eta_{0}=D_{1} \exp \left[\frac{-A_{1}\left(T-T^{*}\right)}{A_{2}+\left(T-T^{*}\right)}\right],
$$

where $D_{1}, A_{1}$, and $A_{2}$ are the fitting parameters and $T^{*}$ is a reference temperature. Table 4 gives the fitting parameters for the Cross-WLF model for this glass-fiber/PP material as reported by Autodesk, Inc. Figure 2 gives the plots of viscosity against strain rate at four temperatures for the studied material.

Table 2 Specific heat $\mathrm{c}_{\mathrm{p}}$ over a range of temperatures $\mathrm{T}$ for the glass-fiber/PP material as reported by Autodesk, Inc. [12].

\begin{tabular}{cc}
\hline \hline \multicolumn{2}{c}{$40 \%$ Weight Glass-fiber/PP } \\
$T\left[{ }^{\circ} \mathrm{C}\right]$ & $C_{p}\left[\mathrm{~J} / \mathrm{kg}-{ }^{\circ} \mathrm{C}\right]$ \\
\hline 260 & 2383.0 \\
135 & 2065.0 \\
125 & 2115.0 \\
121 & 3040.0 \\
118 & 5297.0 \\
115 & 14908.0 \\
112 & 6100.0 \\
109 & 2582.0 \\
100 & 2029.0 \\
75 & 1717.0 \\
60 & 1578.0 \\
50 & 1009.0 \\
\hline
\end{tabular}


Table 3 Thermal conductivity k over a range of temperatures $\mathrm{T}$ for the glass-fiber/PP material as reported by Autodesk, Inc. [12].

\begin{tabular}{cc}
\hline \hline \multicolumn{2}{c}{ 40\% Weight Glass-Fiber/PP } \\
$T\left[{ }^{\circ} \mathrm{C}\right]$ & $k\left[\mathrm{~W} / \mathrm{m}-{ }^{\circ} \mathrm{C}\right]$ \\
\hline 260.6 & 0.197 \\
239.1 & 0.188 \\
218.1 & 0.181 \\
198.4 & 0.188 \\
178.3 & 0.193 \\
157.5 & 0.197 \\
137.1 & 0.174 \\
117.8 & 0.252 \\
97.4 & 0.252 \\
77.2 & 0.232 \\
57.6 & 0.240 \\
38 & 0.259 \\
\hline
\end{tabular}

Table 4 Fitting parameters for the Cross-WLF model for the studied glass-fiber/PP material as reported by Autodesk, Inc. [12].

\begin{tabular}{cc}
\hline \hline Parameter & $\begin{array}{c}\text { 40\% Weight } \\
\text { Glass-Fiber/PP }\end{array}$ \\
\hline$n$ & 0.2768 \\
$\tau^{*}[\mathrm{~Pa}]$ & 35922.1 \\
$\mathrm{D}_{1}[\mathrm{~Pa}-\mathrm{s}]$ & $3.36477 \times 10^{16}$ \\
$T^{*}[\mathrm{~K}]$ & 263.15 \\
$A_{1}$ & 38.390 \\
$A_{2}[\mathrm{~K}]$ & 51.600 \\
\hline
\end{tabular}

\subsection{Correlations between Model Predictions and Experimental Results}

Orientation measurements were performed by ORNL using a Leeds image analysis system developed by Hine et al. [13]. The important orientation descriptors are the orientation tensor elements $A_{11}, A_{22}, A_{33}$, and $A_{31} . A_{11}, A_{22}$, and $A_{33}$ range between 0 and 1. For instance, a high value of $\mathrm{A}_{11}$ at a given point would indicate a great deal of orientation in the flow direction. Similarly, a near-zero value of $A_{33}$ would indicate little or no orientation in the thickness direction (z-direction). On the other hand, $\mathrm{A}_{31}$ ranges between -0.5 and 0.5 . In the flow-thickness direction plane, a value of $\mathrm{A}_{31}$ approaching 0.5 , would indicate high alignment in the direction $45^{\circ}$ to the symmetry plane, whereas a value of $A_{31}$ approaching -0.5 would indicate high alignment in the direction $-45^{\circ}$ to the symmetry plane. 
The ORIENT program assumes symmetry about the mid-plane in the thickness direction, and this is reflected in the orientation predictions: the predicted values of $A_{11}, A_{22}$, and $A_{33}$ are all symmetric about $z=0$, while $A_{31}$ is anti-symmetric about $z=0$. The finite difference mesh used in ORIENT consisted of twenty-one nodes in the thickness direction and 121 nodes in the flow-direction. At each region (A, B, or C), ORIENT predicts the orientation tensor components for twenty-one points in the half-thickness of the piece. For the results discussed in this section, all data have been taken at Region B. At Region A, there is little orientation development beyond the mold inlet, and since the experimental data at Region A was used for determining the inlet boundary condition for the ORIENT calculations, the predictions (not surprisingly) match the experimental data well in this region. In Region $\mathrm{C}$ some samples exhibit significant orientation development beyond Region $\mathrm{B}$, but the qualitative description of the results remains the same as that for Region B.

Figure 3 provides the $A_{11}$ components of the second-order orientation tensor at Region $B$ of the AF3I ISO-plaque as a function of the non-dimensional thickness coordinate $z / b$, where $b$ is the half-thickness of the sample. Experiments show low alignment in the shell (the region near the mold walls) compared to short-fiber thermoplastics, which typically have shell-region $\mathrm{A}_{11}$ values close to 0.8 . Also, a very thick core region (the region near the center of the sample with the lowest flow-direction orientation) was readily apparent. Applying a fiber interaction coefficient of 0.006 (typical for SFTs) to the model results in a poor fit to both the shell-region alignment and the thickness of the core. Increasing the fiber interaction coefficient better captures the shell-region alignment, but does not capture the wide core. Increasing the strain reduction factor and keeping the large $\mathrm{C}_{\mathrm{I}}$ value can reasonably predict the shell-region alignment and the thick core. After several model iterations, it was determined that a fiber interaction coefficient of 0.03 combined with a SRF>1 within the RSC model provided superior predicted results.

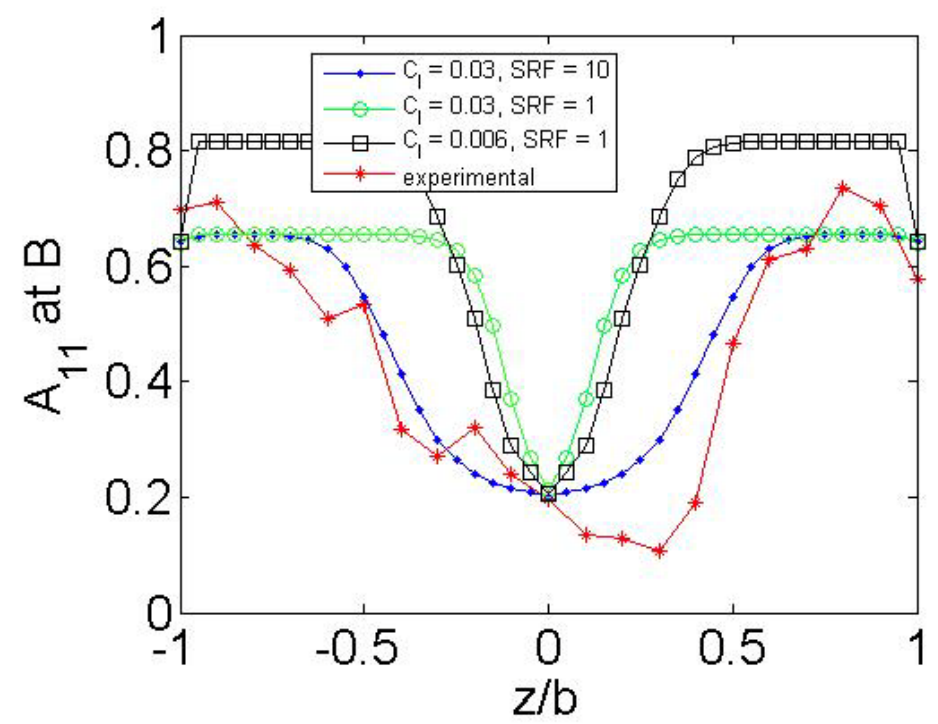

Figure $3 A_{11}$ vs. non-dimensional thickness coordinate $z / b$ for the AF3I ISO-plaque (Table 1). The experimental data is compared against three different sets of parameters for orientation modeling. The best fit is provided by the RSC model with a $S R F$ factor much greater than one and $C_{\mathrm{I}}=0.03$. 


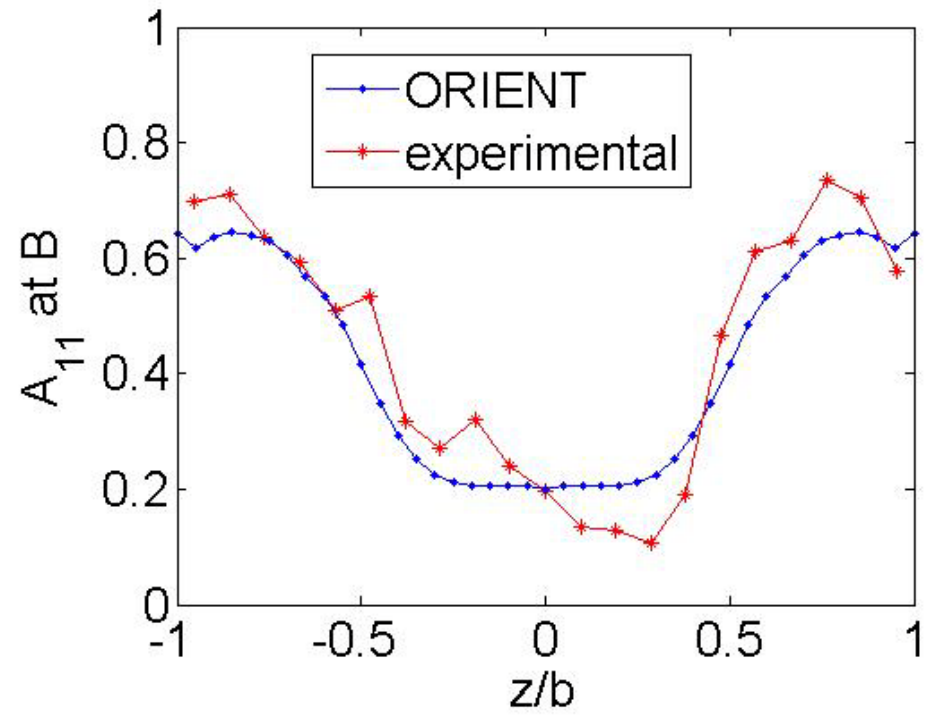

Figure $4 A_{11}$ vs. non-dimensional thickness coordinate $z / b$ for AF3I at Region B. $S R F=30$ and

$$
C_{\mathrm{I}}=0.03 \text {. }
$$

Implementing the RSC model with an SRF factor of 30 and keeping $C_{I}=0.03$ provides a good fit of the $\mathrm{A}_{11}$ tensor component for all glass fiber samples considered, regardless of fill speed or mold geometry. Thus, one set of fitting parameters are sufficient for each of the glass fiber-reinforced moldings in these trials. This is a significant finding resulting from our work. Figures 4-7 illustrate the $\mathrm{A}_{11}$ tensor component at Region $\mathrm{B}$ for all glass fiber samples. However, using the parameters described previously under-predicts the value of $A_{22}$. By extension, $A_{33}$ is over-predicted, since the trace of $\mathbf{A}$ equals unity. As an example, Figure 8 gives $\mathrm{A}_{22}$ and $\mathrm{A}_{33}$ for a slow-filled glass fiber ISO plaque (AS3I). In general, $\mathrm{A}_{31}$ is poorly fit for all samples considered.

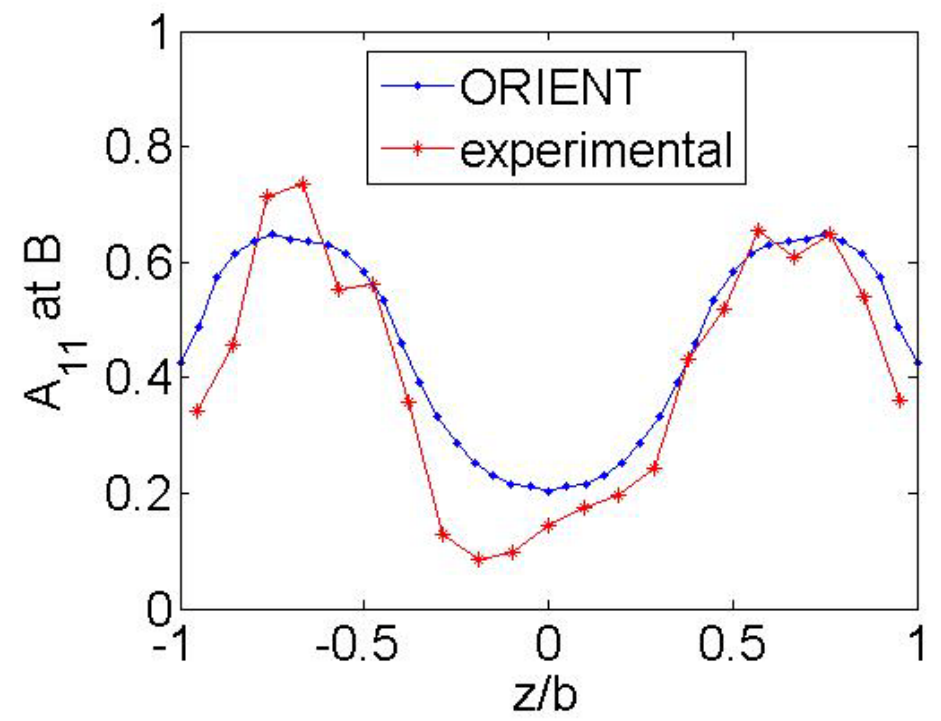

Figure $5 A_{11}$ vs. non-dimensional thickness coordinate $z / b$ for AS3I at Region B. $S R F=30$ and

$$
C_{\mathrm{I}}=0.03 \text {. }
$$




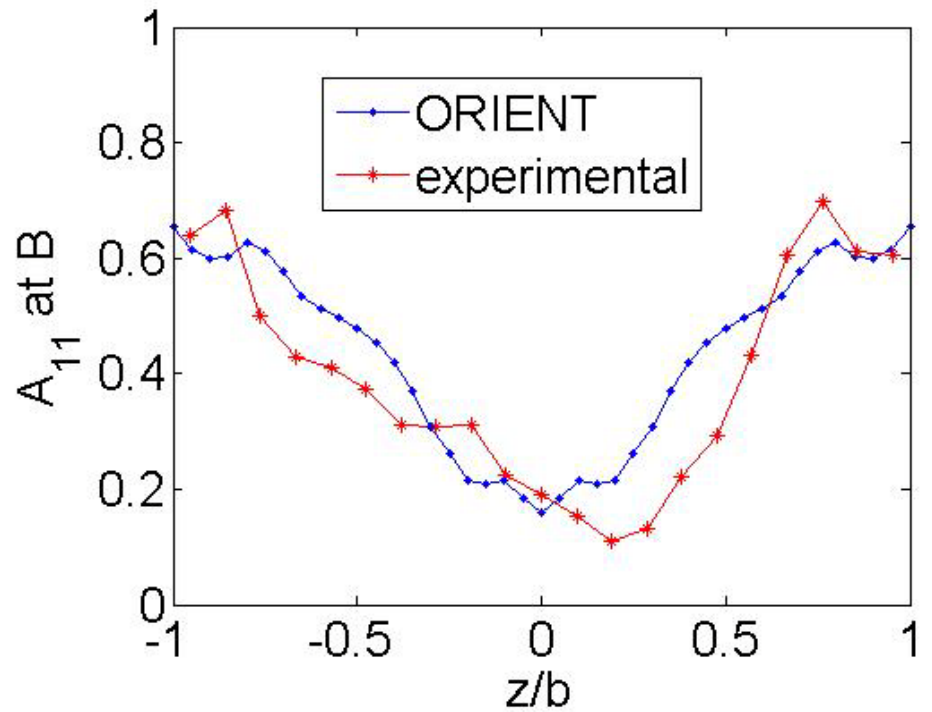

Figure $6 A_{11}$ Vs. non-dimensional thickness coordinate $z / b$ for AF3D at Region B. $S R F=30$ and $C_{\mathrm{I}}=0.03$.

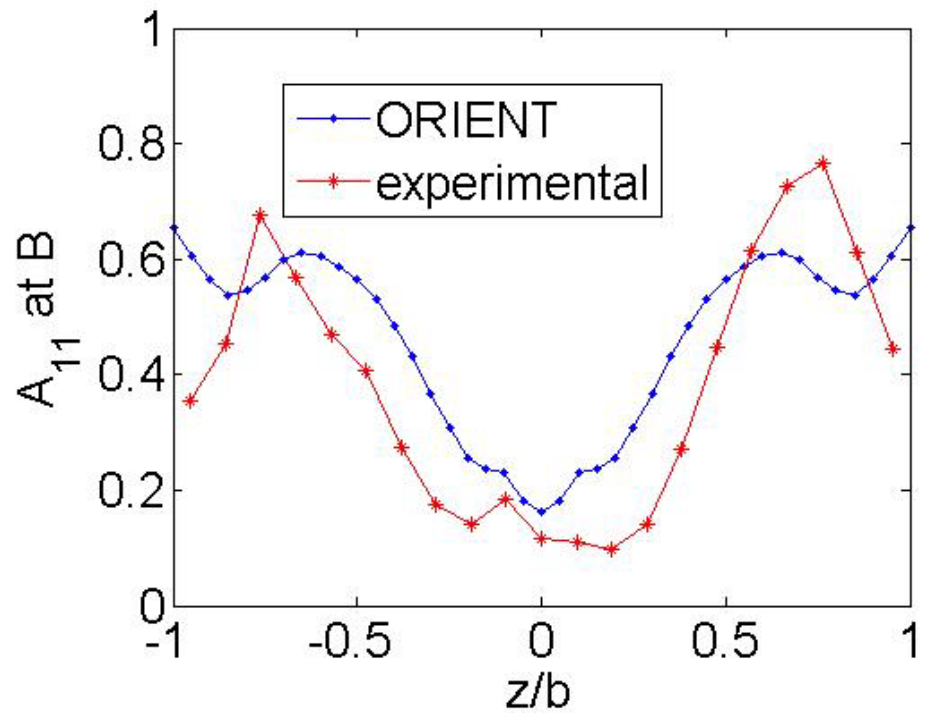

Figure $7 A_{11}$ vs. non-dimensional thickness coordinate $z / b$ for AS3D at Region B. $S R F=30$ and $C_{\mathrm{I}}=0.03$. 


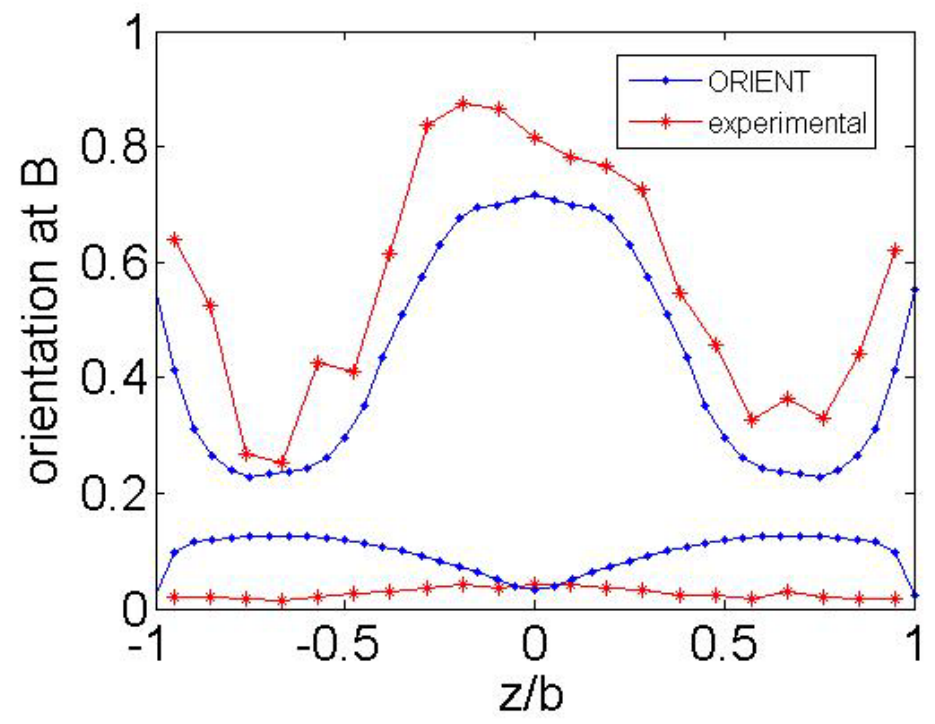

Figure $8 A_{22}$ and $A_{33}$ vs. non-dimensional thickness coordinate $z / b$ for AS3I at Region B. SRF = 30 and $C_{I}=0.03$.

The assessment of the fiber orientation models developed for SFTs shows that the RSC model is a significant improvement of the Folgar-Tucker model to address the fiber orientation in LFTs. However, only qualitative agreement has been found with the experimental results measured with the Leeds system. Neither the Folgar-Tucker nor the RSC models can predict fiber orientation in LFT samples to a level of accuracy needed for predictive engineering. The fundamental limitation of the fiber orientation model resides in the interaction term. This term needs to be improved to account for the rotation of long fibers and the anisotropic character of the fiber-fiber interaction which cannot be adequately represented by an isotropic rotary diffusion term. To address this issue, the University of Illinois team has developed a new fiber orientation model for LFTs that incorporates an anisotropic rotary diffusion term. Section 4 provides the description of this model that is termed the anisotropic rotary diffusion reduced strain closure (ARD-RSC) model [5-6].

\subsection{The Anisotropic Rotary Diffusion - Reduced Strain Closure Model}

The Folgar-Tucker model accounts for fiber-fiber interactions through the isotropic rotary diffusion governed by $C_{\mathrm{I}}[1-2]$. Phelps and Tucker suggest that accounting for such interactions with the anisotropic rotary diffusion (ARD) should allow us to better capture the fiber orientation distribution in LFTs. Such a model then replaces the scalar $C_{\mathrm{I}}$ with a tensorial description $\mathbf{C}$ for the fiber-fiber interactions.

Previously, Phan-Thien and Fan [14] proposed a fiber orientation model using anisotropic rotary diffusion. However, the Phan-Thien/Fan model's diffusion term failed to return the fibers to an isotropic orientation at steady state, a necessary condition of any diffusion model. To correct the Phan-Thien/Fan model, an expression for rotary diffusion was developed that was 
defined on the surface of the unit sphere traced by all orientations of the unit vector $\mathbf{p}$ [5-6]. The expression for the ARD model to properly match the LFT fiber orientation data is:

$$
\begin{aligned}
& \dot{\mathbf{A}}=(\mathbf{W} . \mathbf{A}-\mathbf{A} \cdot \mathbf{W})+\xi(\mathbf{D} . \mathbf{A}+\mathbf{A} \cdot \mathbf{D}-2 \overline{\overline{\mathbf{A}}}: \mathbf{D}) \\
& +\dot{\gamma}(2 \mathbf{C}-2(\operatorname{tr} \mathbf{C}) \mathbf{A}-5(\mathbf{C} \cdot \mathbf{A}+\mathbf{A} \cdot \mathbf{C})+10 \overline{\overline{\mathbf{A}}}: \mathbf{C})
\end{aligned}
$$

where $\mathbf{A}$ and $\overline{\overline{\mathbf{A}}}$ are the second- and fourth-order orientation tensors, respectively. $\dot{\mathbf{A}}=D \mathbf{A} / D t$ with $t$ being the time. $\mathbf{W}$ is the vorticity tensor, and $\mathbf{D}$ is the rate of the deformation tensor. $\dot{\gamma}$ is the scalar magnitude of $\mathbf{D}$, and $\xi$ is the shape parameter ( $\xi=1$ for any fiber). Tensor $\mathbf{C}$ is constructed from the $\mathbf{A}$ and $\mathbf{D}$ tensors as:

$$
\mathbf{C}=b_{1} \mathbf{I}+b_{2} \mathbf{A}+b_{3} \mathbf{A}^{2}+b_{4} \frac{\mathbf{D}}{\dot{\gamma}}+b_{5} \frac{\mathbf{D}^{2}}{\dot{\gamma}^{2}}
$$

where $b_{i}(i=1, . ., 5)$ are the scalar constants. A systematic method of selecting $b_{i}$ was developed in [5-6] to ensure stable and valid orientation solutions. To properly match experimental orientation data, it is necessary to slow the predicted orientation dynamics of a given model. For instance, the RSC model [3] can objectively slow the orientation dynamics of the Folgar-Tucker model. Treating the ARD model similarly, the ARD-RSC model is obtained as:

$$
\begin{aligned}
& \dot{\mathbf{A}}^{\mathrm{ARD}-\mathrm{RSC}}=(\mathbf{W} \cdot \mathbf{A}-\mathbf{A} \cdot \mathbf{W}) \\
& +\xi(\mathbf{D} \cdot \mathbf{A}+\mathbf{A} \cdot \mathbf{D}-2[\overline{\overline{\mathbf{A}}}+(1-\kappa)(\overline{\overline{\mathbf{L}}}-\overline{\overline{\mathbf{M}}}: \overline{\overline{\mathbf{A}}})]: \mathbf{D}) \\
& +\dot{\gamma}(2[\mathbf{C}-(1-\kappa) \overline{\overline{\mathbf{M}}}: \mathbf{C}]-2 \kappa(\operatorname{tr} \mathbf{C}) \mathbf{A} \\
& -5(\mathbf{C} \cdot \mathbf{A}+\mathbf{A} \cdot \mathbf{C})+10[\overline{\overline{\mathbf{A}}}+(1-\kappa)(\overline{\overline{\mathbf{L}}}-\overline{\overline{\mathbf{M}}}: \overline{\overline{\mathbf{A}}})]: \mathbf{C})
\end{aligned}
$$

where $\mathbf{C}$ is given by Equation (6). $\overline{\overline{\mathbf{L}}}$ and $\overline{\overline{\mathbf{M}}}$ are the fourth-order tensors that are defined in terms of the eigenvalues and eigenvectors of $\mathbf{A} . \kappa(<1)$ is a scalar parameter which controls the rate of orientation dynamics.

The ARD-RSC model was implemented in ORIENT and was then used in the injection-molding analyses of the glass-fiber/PP samples presented in the previous section. With $\kappa=1 / 30$ and $b_{1}=$ $7.848 \times 10^{-4}, b_{2}=2.357 \times 10^{-2}, b_{3}=1.0 \times 10^{-2}, b_{4}=1.168 \times 10^{-5}$, and $b_{5}=-3.0 \times 10^{-3}$, an excellent fit to all the experimental orientation data was obtained for the AF3I ISO-plaque (Figures 9 and 10). Very good correlations with the experimental orientation data were also obtained for the other samples (disk or plaque). 


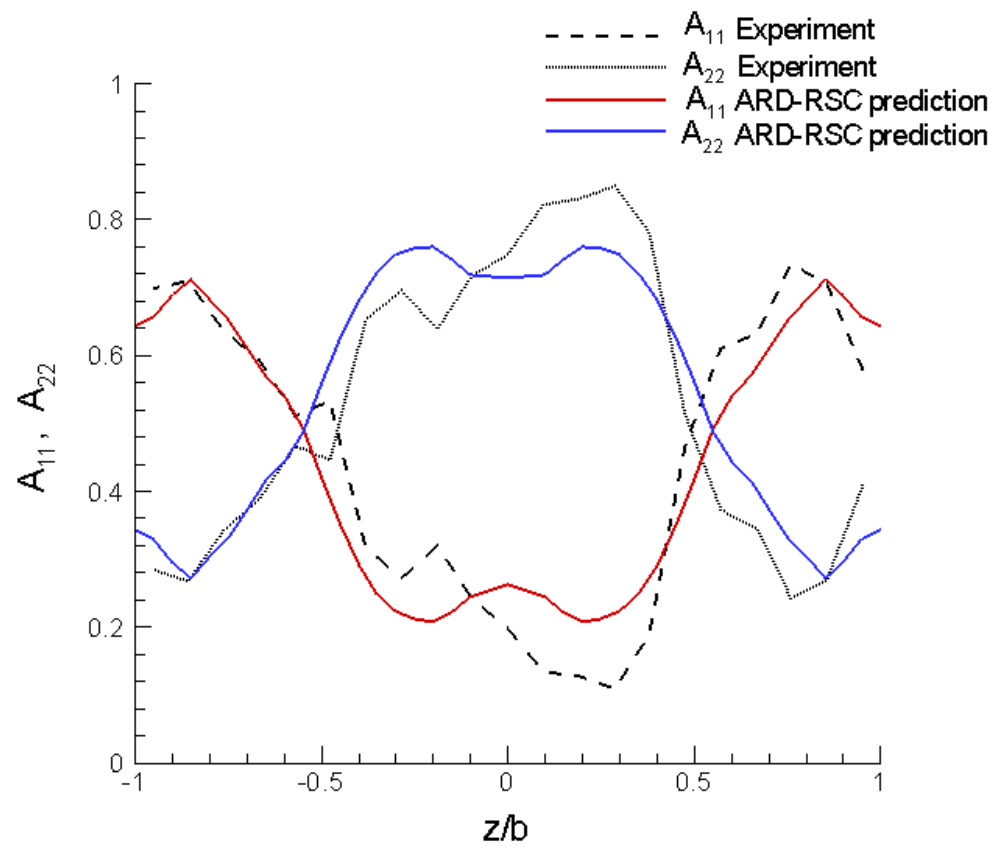

Figure 9 Predicted (by the ARD-RSC model implemented in ORIENT) and measured fiber orientation tensor components $A_{11}$ and $A_{22}$ for Region B of the AF3I ISO-plaque.

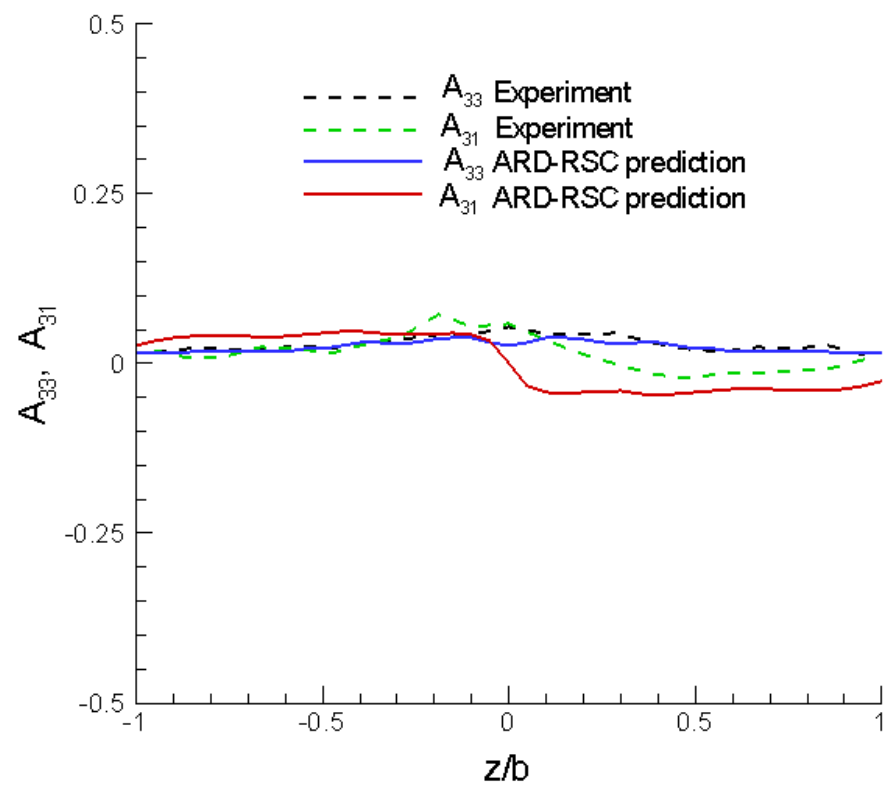

Figure 10 Predicted (by the ARD-RSC model implemented in ORIENT) and measured fiber orientation tensor components $A_{33}$ and $A_{31}$ for Region B of the AF3I ISO-plaque. 


\subsection{Development of A Fiber Length Attrition Model in the Mold}

Phelps and Tucker [6-7] have developed a fiber length attrition model to predict fiber length distribution in a mold cavity during injection molding. First, using a model by Dinh and Armstrong [15], an expression for the hydrodynamic force acting along the fiber axis is obtained. The condition for buckling that leads to fiber breakage compares this hydrodynamic force to the buckling force from the classical Euler buckling theory. This condition states that a fiber of length $l_{i}$ and orientation $\mathbf{p}$ will break if

$$
\frac{F_{i}(\mathbf{p})}{F_{\text {crit }}}=\left[\frac{4 \zeta \eta_{\mathrm{m}} l_{i}^{4}}{\pi^{3} E_{\mathrm{f}} d_{\mathrm{f}}^{4}}\right](-2 \mathbf{D}: \mathbf{p p})>1
$$

Here $F_{\text {crit }}$ is the critical compressive force based on the Euler buckling theory, $\zeta$ a dimensionless drag coefficient from the Dinh-Armstrong model, $\eta_{\mathrm{m}}$ the resin viscosity, $\mathbf{D}$ the rate of the deformation tensor, and $E_{\mathrm{f}}$ and $d_{\mathrm{f}}$ are the fiber elastic modulus and diameter, respectively.

Using criterion (8) in combination with typical orientation statistics of fibers, Phelps and Tucker express the probability that a fiber of length $l_{i}$ will break during a time increment $\Delta t$ as $P_{i} \Delta t$, where $P_{i}$ is given by

$$
P_{i}=C_{B} \dot{\gamma}\{1-\exp (1-\hat{\dot{\gamma}})\}
$$

where $C_{B}$ is a phenomenological coefficient that scales the breakage rate, and $\hat{\dot{\gamma}}$ is the expression in square brackets in Equation (8).

The local fiber length distribution is represented by a set of values $N_{i}, i=1$ to $n$, that give the number of fibers of length $l_{i}=i \Delta l$. Typically $n=130$ bins is used in the length distribution. As fibers break, this distribution must satisfy an equation expressing conservation of the total fiber length

$$
\frac{\partial N_{i}}{\partial t}+\mathbf{v} \bullet \nabla N_{i}=-P_{i} N_{i}+\sum_{k} R_{i k} N_{k}
$$

In this equation $R_{i k}$ is the rate of production of child fibers of length $l_{i}$ by breaking parents of length $l_{k}$ and $\mathbf{v}$ is the fluid velocity. $R_{i k}$ is determined by a combination of the parent breakage rate $P_{k}$ and the assumption that breaking points are distributed along the parent fiber length in a Gaussian profile. Together with Equations (8)-(10) this provides a full set of equations to solve for the fiber length distribution.

This fiber length attrition model has been implemented in ORIENT to enable fiber length predictions for injection molded LFT parts. ORIENT provides local values of viscosity, velocity

and shear rate. Each node in the filling mesh has a length distribution (a set of $N_{i}$ values). The fiber length distribution is carried along with the polymer as it fills the mold cavity, and changes 
as the fibers break in response to the local shear rate. The measured fiber length distribution just inside the gate is used as an initial condition, and the model predicts the length distribution at all downstream locations.

Figure 11 shows measured and predicted fiber length distributions for the glass-fiber/PP disk (AF3D). The experimental length distribution is typical of samples with a significant amount of fiber length attrition: the majority of the fibers are less than $2 \mathrm{~mm}$ long, but some longer fibers remain. The predictions of this fiber length attrition model capture the features of this data very well. The change of average fiber length along the flow path is shown in Figure 12 for this same glass-fiber/PP molding. The plot shows number-average $\left(L_{n}\right)$ and weight-average $\left(L_{w}\right)$ fiber lengths as a function of distance from the injection point. Again, the model accurately predicts these changes.

Future work with this model will include a more comprehensive comparison of predicted and measured fiber length data. An evolution equation will be developed for average fiber length measures, which are more convenient for inclusion in production mold-filling simulation software.

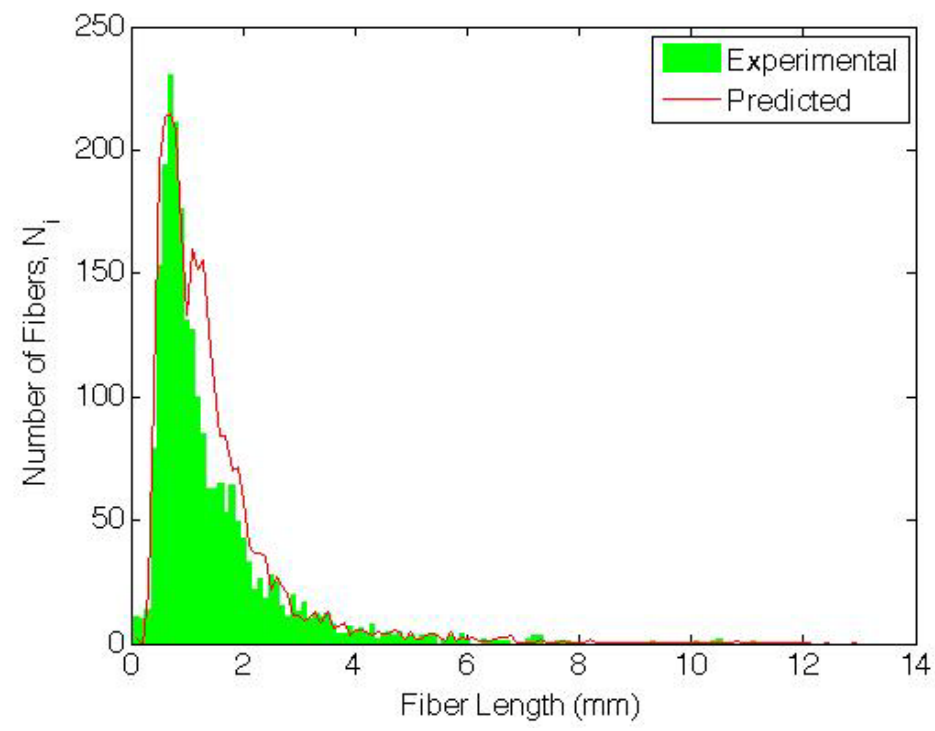

Figure 11 Measured number of fibers vs. fiber length for a glass-fiber/PP disk (AF3D), compared to predictions of the fiber length model. 


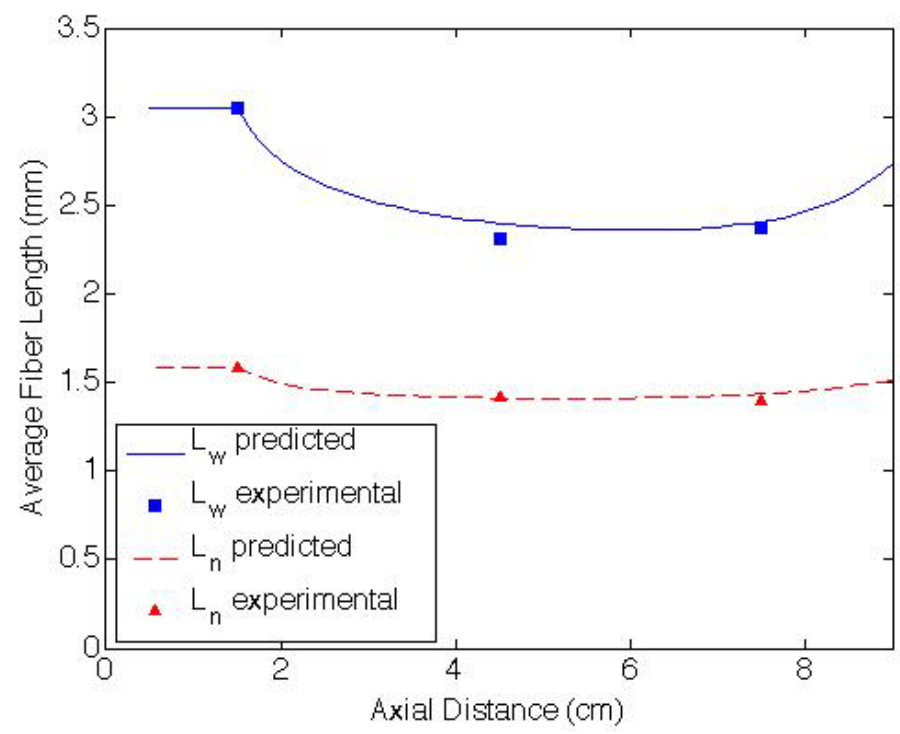

Figure 12 Predicted and experimental average fiber lengths for the same glass-fiber/PP disk shown in Figure 11.

\subsection{Implementations of New Process Models in Autodesk Moldflow Insight}

Autodesk, Inc. (formerly Moldflow Corporation) as a major participant of this CRADA (Nr. 260) has worked with PNNL for the last three years or so, on long fiber orientation and fiber length breakage predictions for injection molding of long-fiber thermoplastics. During the contract period, Autodesk, Inc. has completed the implementations of the long fiber orientation and long fiber breakage models by UIUC. Autodesk, Inc. also provided material testing services for long fiber materials. It shipped the original and special builds of the Autodesk Moldflow Insight software at different stages to PNNL. Technical supports of Autodesk Moldflow Insight were also provided to PNNL by Autodesk, Inc. These in-kind contributions from Autodesk, Inc. to the long fiber thermoplastics (LFT) project did not result in any cost for the US Department of Energy (DOE).

In detail, Autodesk, Inc. has performed the following tasks during the contract period:

- Provided technical research and development staff time to support the research effort in developing a new fiber orientation distribution (FOD) model and fiber length attrition model to predict fiber length distribution (FLD).

- Implemented the new FOD model, namely the anisotropic rotary diffusion - reduced strain closure (ARD-RSC) models, developed by Phelps, Tucker, and Wang in both the mid-plane/dual domain and three-dimensional (3D) versions of the Autodesk Moldflow Insight solvers.

- Implemented the fiber length attrition model developed by Phelps and Tucker in both the mid-plane/dual domain and 3D versions of the Autodesk Moldflow Insight solvers. 
- Provided material property testing services and data values stored in Moldflow Standard Material Database ready to use for a number of long fiber filled materials.

- Provided Moldflow software, both original release and special builds with various implementation stages, to PNNL and technical support on using the software.

- Provided documents on the implementations.

\subsection{Fiber Orientation Distribution Model Implementations}

The RSC and ARD-RSC models have been implemented in a research version of Autodesk Moldflow Insight in the mid-plane/dual domain and 3D solvers. The implementation of the mathematical models in the fiber solvers is straightforward, but a couple of issues associated with the ARD-RSC model have first been solved to guarantee the accuracy of predictions.

\section{Inlet Orientation:}

Due to the nature of slow orientation kinetics of the RSC model, when using a typical value of the RSC factor for injection molding simulations, the fiber orientation does not change much along the flow length in some simple geometries such as the end-gate plaque and center-gated disk [16]. In addition, the orientation in the middle of a part strongly depends on the inlet orientation at the gate. An appropriate inlet orientation condition is crucial to obtain accurate orientation predictions in the part.

An inlet orientation condition can be prescribed using the measured fiber orientation values near the gate. The Autodesk Moldflow Insight Mid-plane and Dual Domain solver provides several options for the inlet orientation profiles across the thickness. The default option has been modified to match the experimental data measured near the gate as illustrated in Figure 13 for the end-gated plaque molded with long-fiber materials. One option has also been added to allow users to specify the inlet fiber orientation which may be different from the default one because of a different geometry or a different material. In the Autodesk Moldflow Insight 3D solver, a similar inlet orientation profile is defined in a small region near the gate if the injection location is situated directly on the part or within the interface between the beam and the part if an existing runner system is modeled by beam elements.

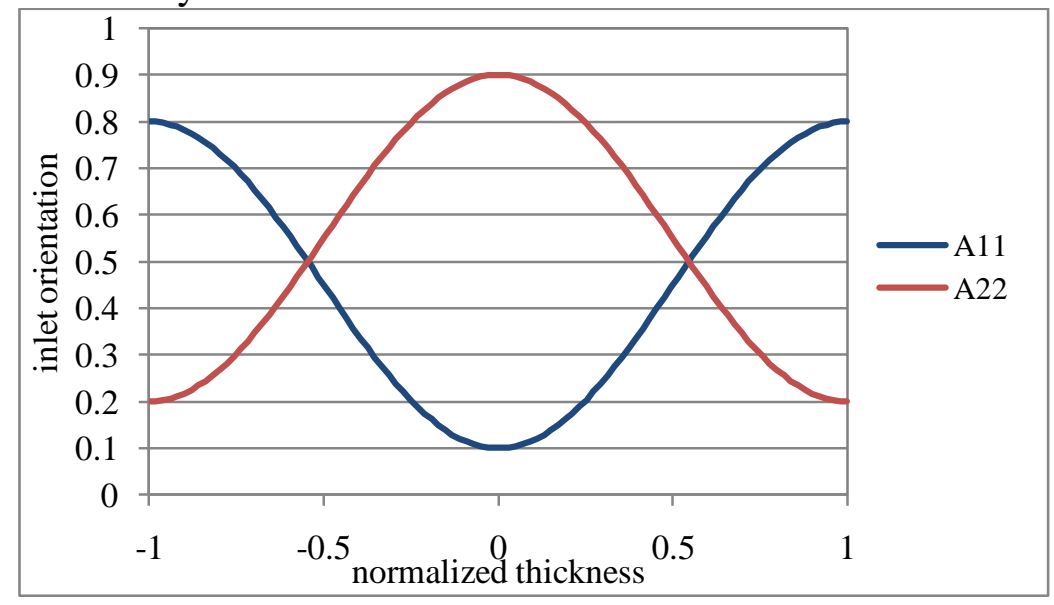

Figure 13. The default inlet orientation to match the long-fiber orientation data. 


\section{Mesh Sensitivity Analysis}

The ARD-RSC model implemented in a previously provided special build of the Autodesk Moldflow Insight mid-plane/dual domain solver presented a mesh dependency problem as shown in Figure 14. The fiber orientation prediction by the ARD-RSC model did not converge, especially at the middle of the thickness, with the mesh refinement. The difference in results between two consecutive refinements was about 10\%. However, the standard Folgar-Tucker model does not suffer from such a problem. This problem was due to the inaccurate computation of the velocity gradient involved in the fiber orientation calculation. In the standard fiber orientation model, the fiber orientation is quickly changed by the velocity gradient and reaches the steady state at small strain; thus the orientation in the middle of the final part, which corresponds to a large strain, is almost the same although the final strains are different in different meshes. However, the orientation predicted by the ARD-RSC model in the final part did not reach the steady state. This resulted in different fiber orientations in different meshes because of different strains in these meshes. Therefore, the schemes for the gradient calculations have been modified to improve the accuracy of the velocity gradient and orientation. Figure 15 shows the results after the change that shows good convergence of the fiber orientation prediction with the mesh refinement.

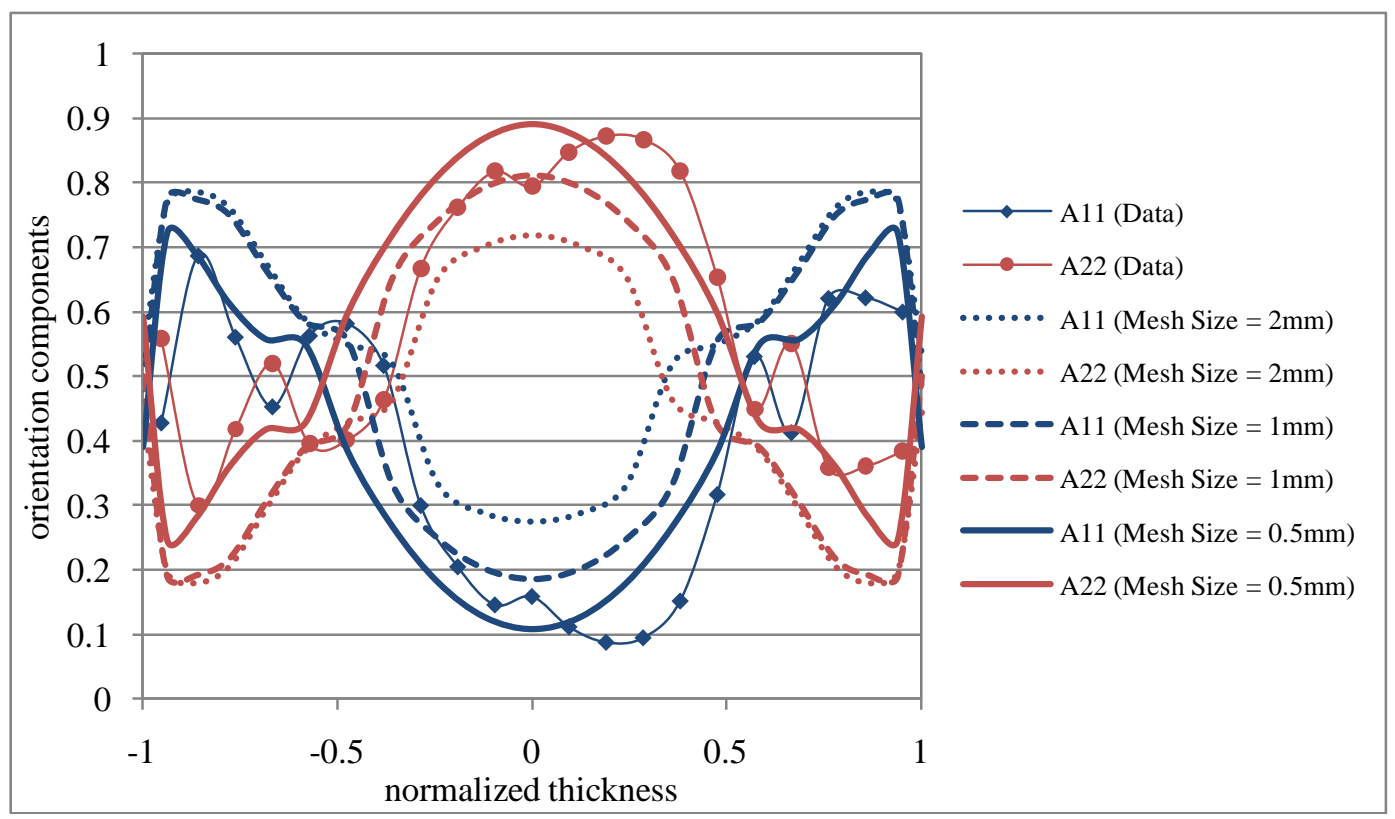

Figure 14 Fiber orientations predicted by the ARD-RSC model with $\kappa=0.05$ for an end-gated plaque modeled with different mid-plane mesh sizes. The results are obtained from the previous special build prior to the gradient modification of Autodesk Moldflow Insight. 


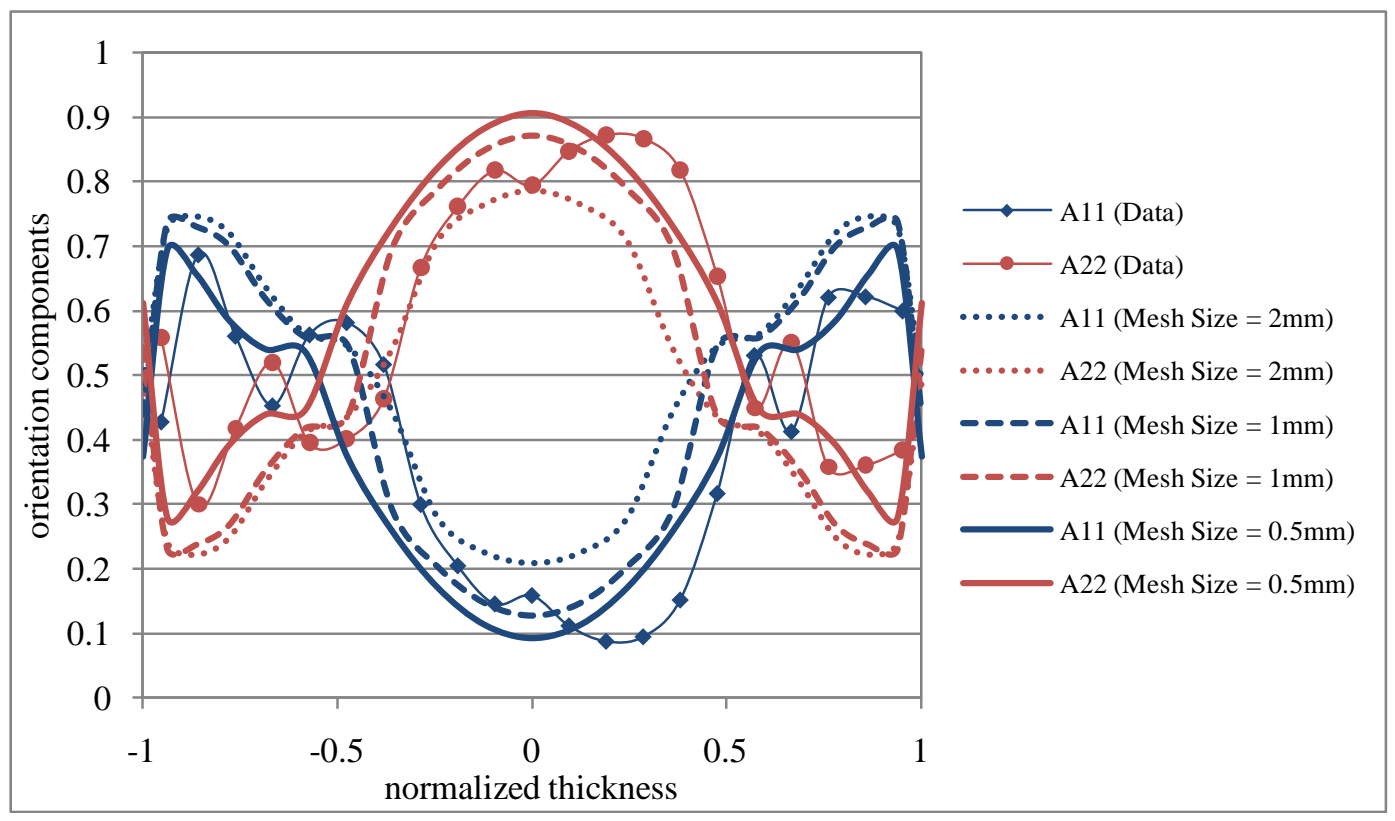

Figure 15 Fiber orientations predicted by the ARD-RSC model with $\kappa=0.05$ for an end-gated plaque modeled with different mid-plane mesh sizes. The results are obtained from the special build with the modification of the gradient calculations.

\section{Comparison with Experimental Data}

The RSC and ARD-RSC models implemented in Autodesk Moldflow Insight were used to simulate the injection molding to predict the fiber orientations in the glass-fiber/PP samples presented in Section 3 (see Figure 1 and Table 1). Both mid-plane and 3D models were used in the Autodesk Moldflow Insight simulations. The meshes are displayed in Figures 16 and 17 for the mid-plane modeling and in Figures 18 and 19 for the 3D modeling. The ARD-RSC model with $\kappa=1 / 30$ was applied to predict the fiber orientation in the parts.

The Autodesk Moldflow Insight mid-plane results show good agreement with the experimental data in all the studied samples. The results are presented in Figures 20 to 23. The inlet orientation shown in Figure 13 was applied at the gate. Autodesk Moldflow Insight predicts a wide core region and also predicts reasonably good fiber orientation in the shell layers.

However, the Autodesk Moldflow Insight 3D results do not agree with the experimental data. The predictions shown in Figures 24 to 27 give an almost random core and do not match the transversely aligned core as observed in experiments. Overall, the $A_{11}$ component is too large and the $A_{22}$ component is too small compared to the experimental data. The reason for the large discrepancy between the prediction and the data is that the inlet orientation at the gate is not carried along with the flow. The prescribed inlet orientation is applied at a small region around the gate and the complex 3D flow entering the gate quickly changes the orientation. In the 3D solver, the inlet orientation has a small influence on the orientation in the middle of part. A more appropriate method to apply the inlet in 3D is highly desired and will be sought by Autodesk, Inc. 


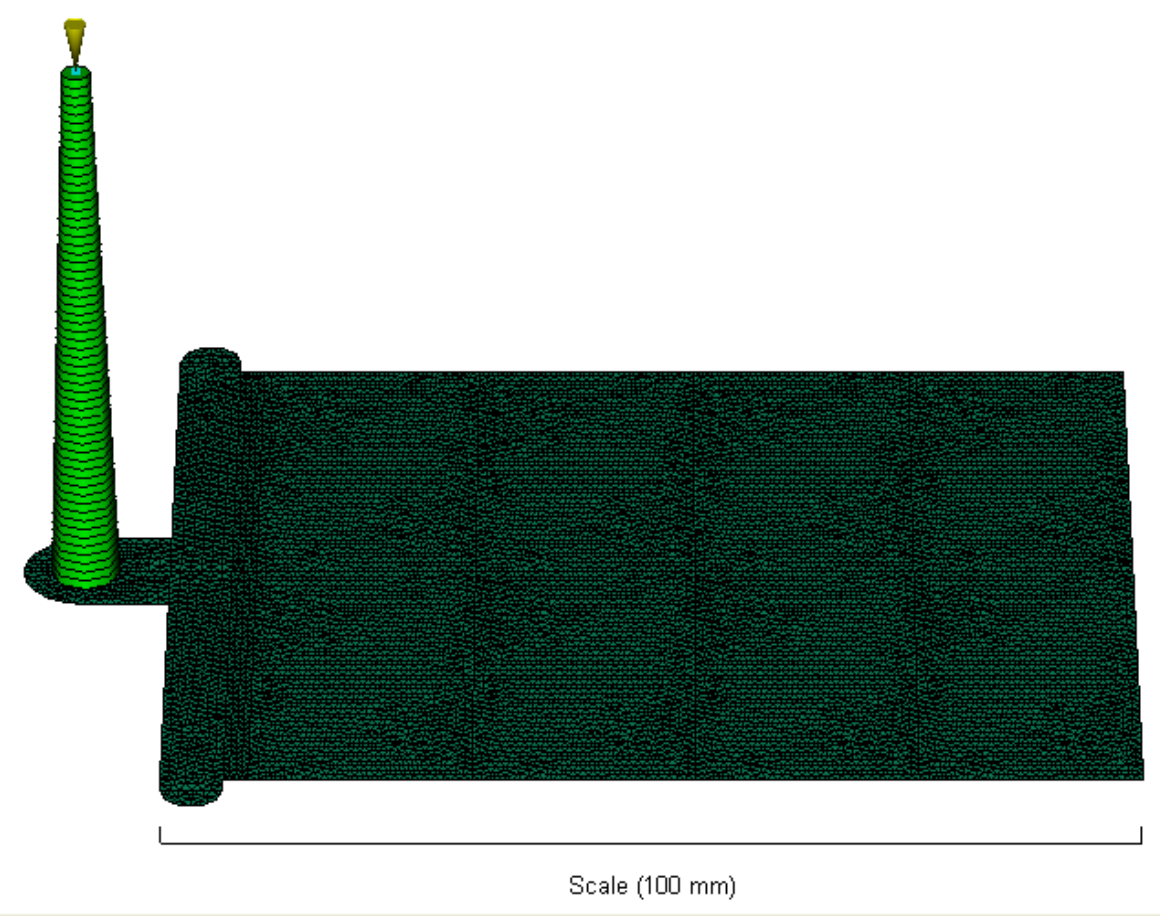

Figure 16 A three-mm thick end-gated plaque (AF3I or AS3I) modeled by a mid-plane mesh.

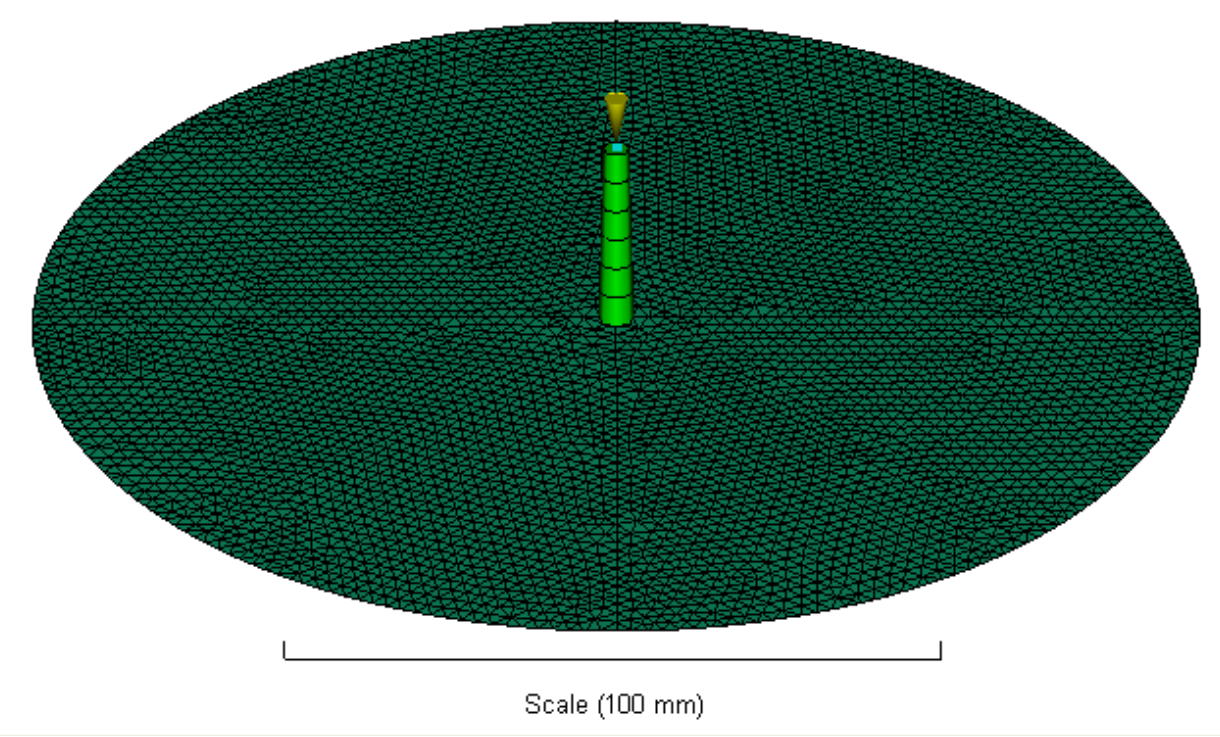

Figure 17 A three-mm thick center-gated disk (AF3D or AS3D) modeled by a mid-plane mesh. 


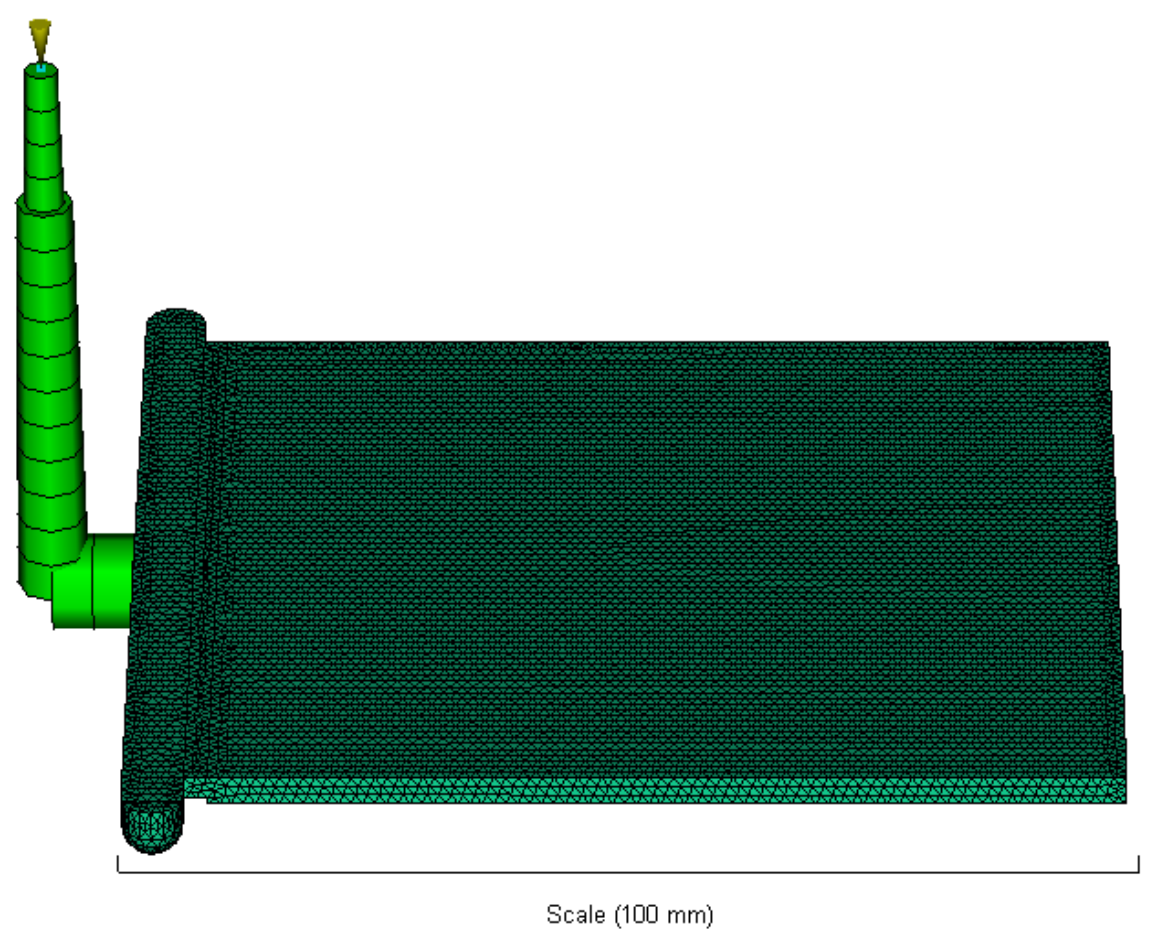

Figure 18 A three-mm thick end-gated plaque (AF3I or AS3I) modeled by a 3D mesh.

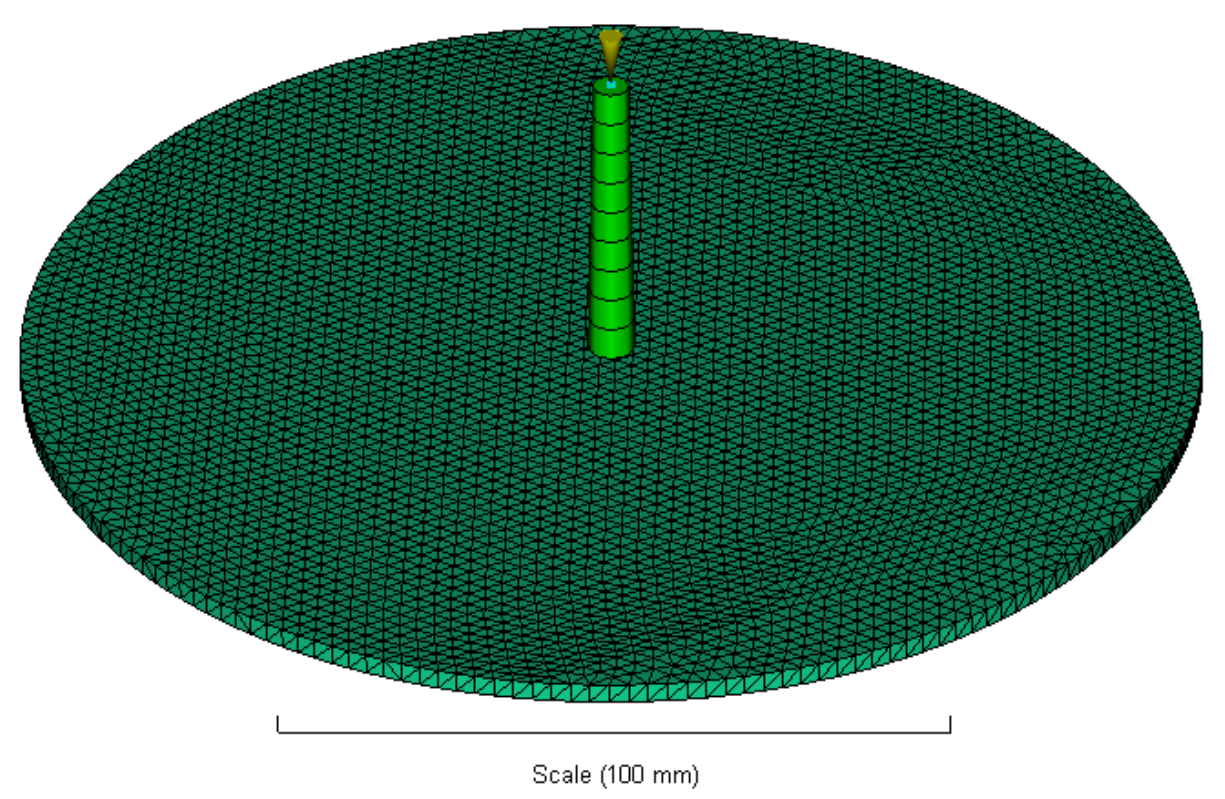

Figure 19 A three-mm thick center-gated disk (AF3D or AS3D) modeled by a 3D mesh. 

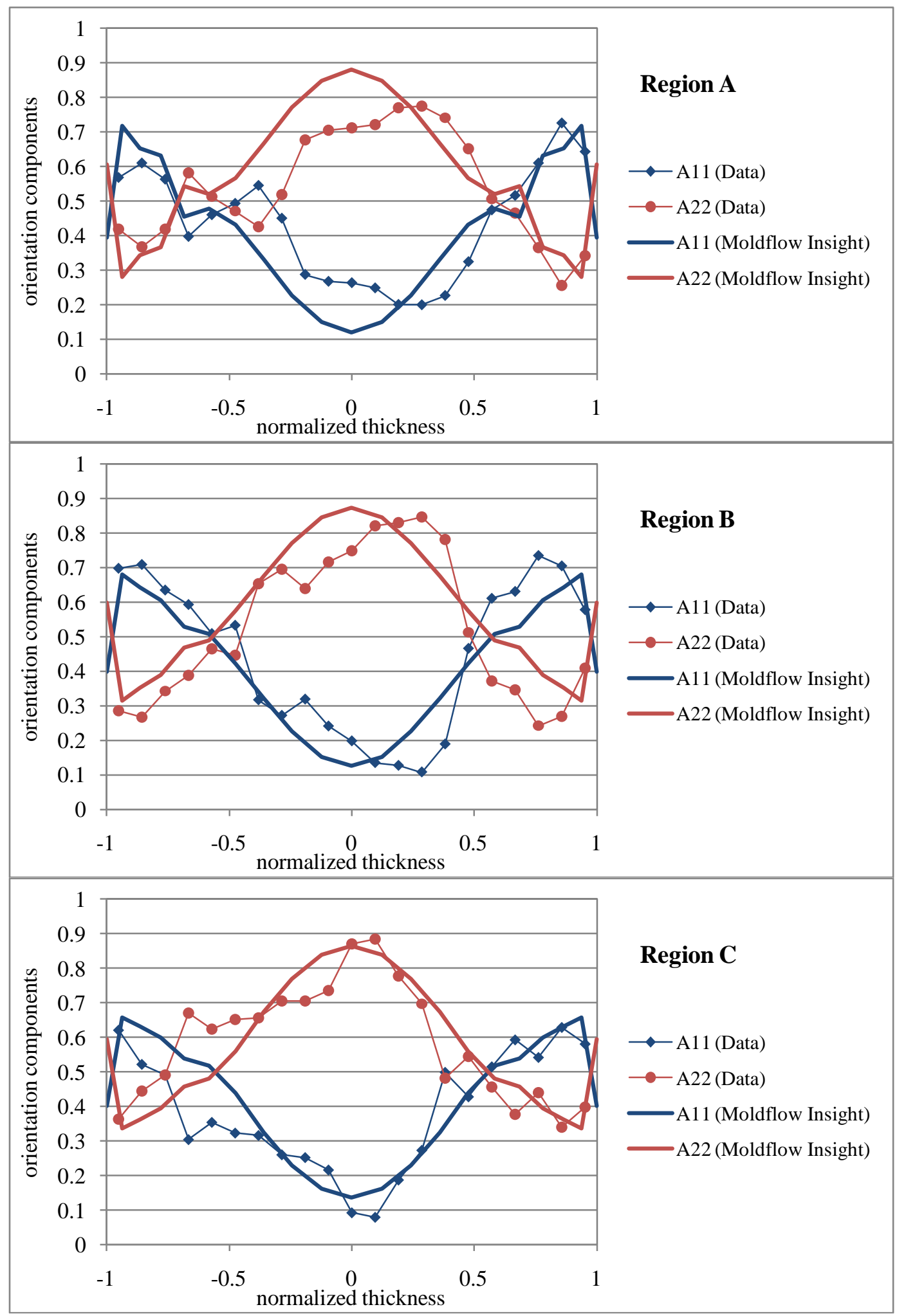

Figure 20 Comparison of fiber orientation data with the prediction by the ARD-RSC model with $\kappa=1 / 30$ for the AF3I ISO-plaque (fast fill) using a mid-plane mesh. 


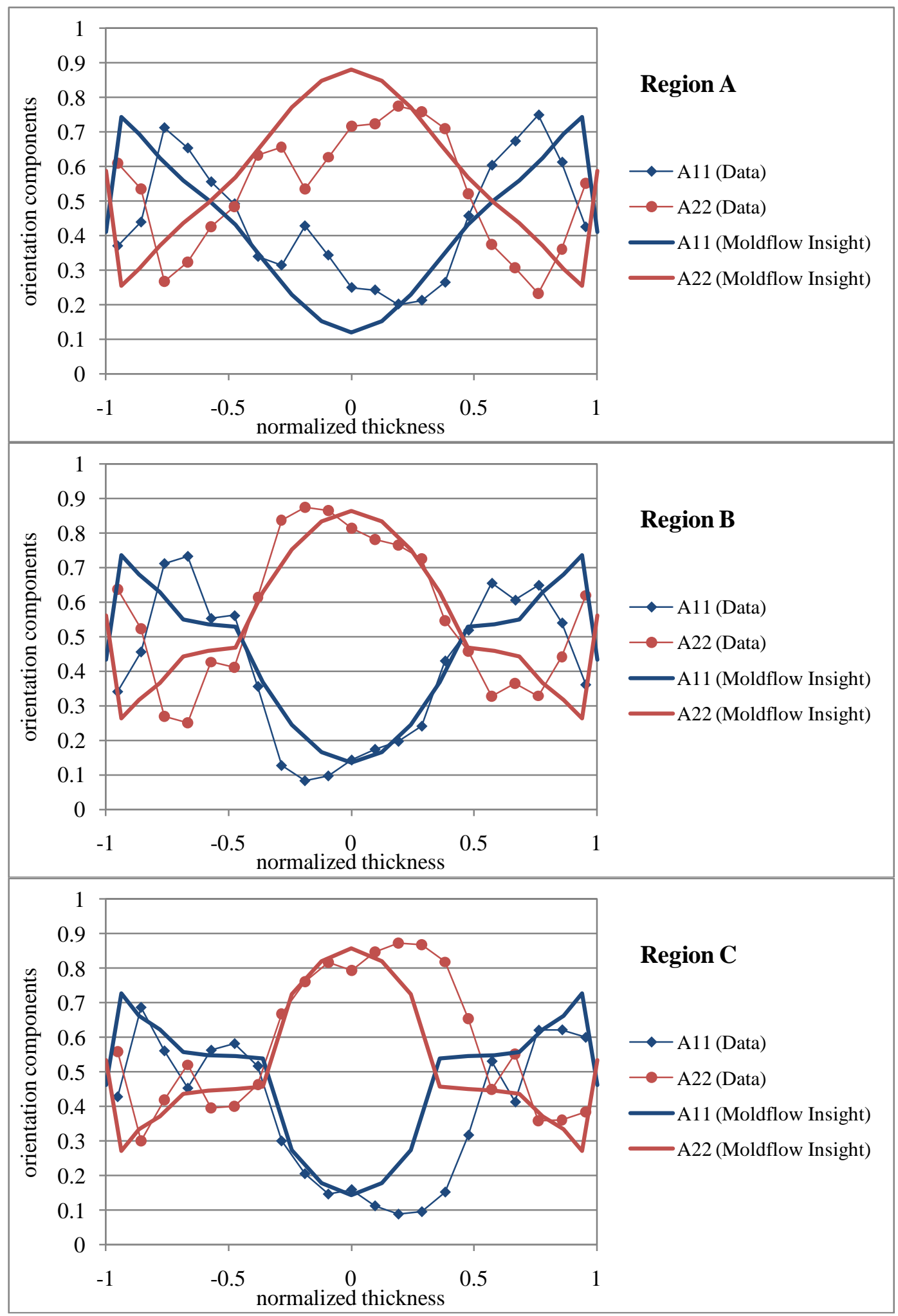

Figure 21 Comparison of fiber orientation data with the prediction by the ARD-RSC model with $\kappa=1 / 30$ for the AS3I ISO-plaque (slow fill) using a mid-plane mesh. 


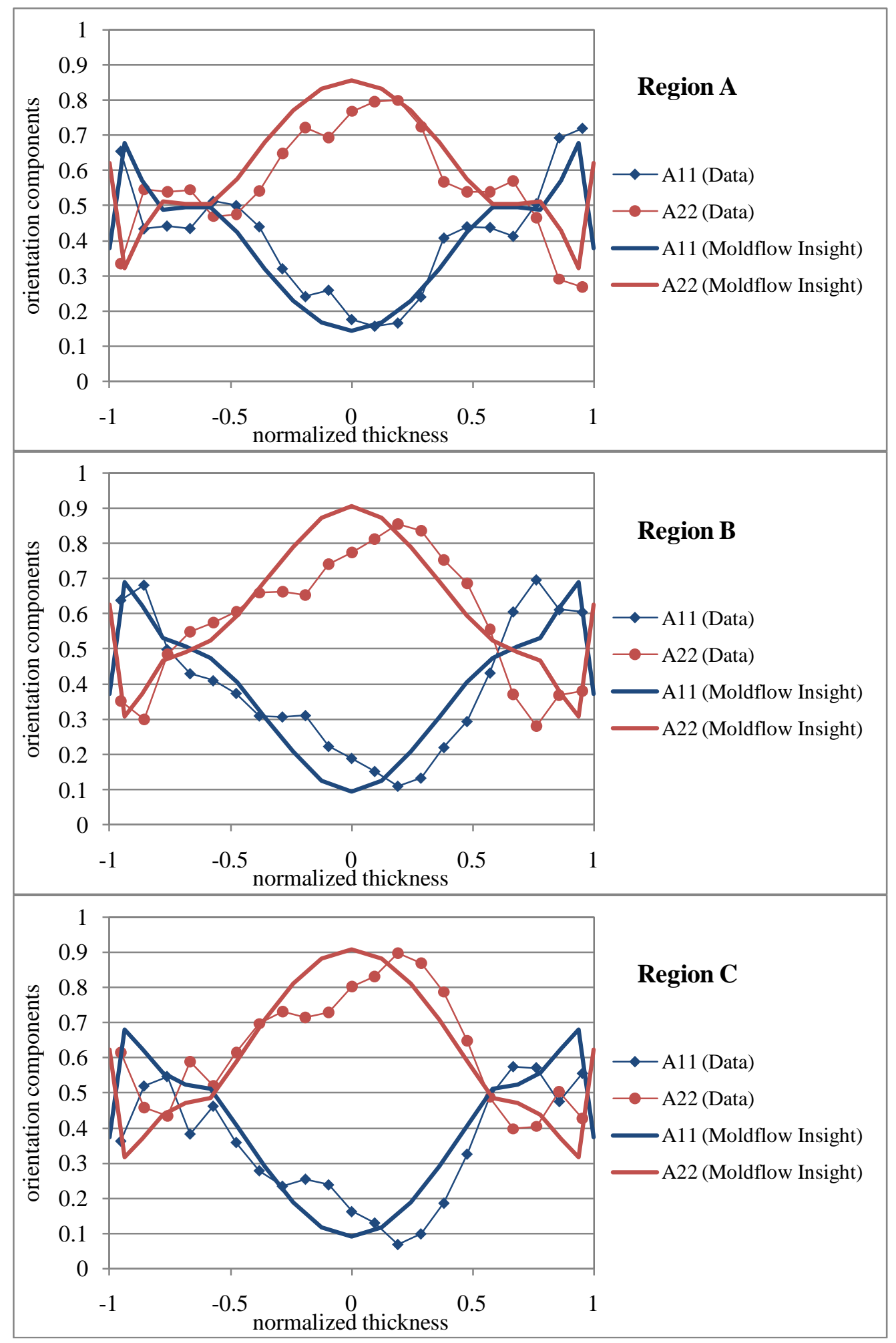

Figure 22 Comparison of fiber orientation data with the prediction by the ARD-RSC model with $\kappa=1 / 30$ for the AF3D disk (fast fill) using a mid-plane mesh. 


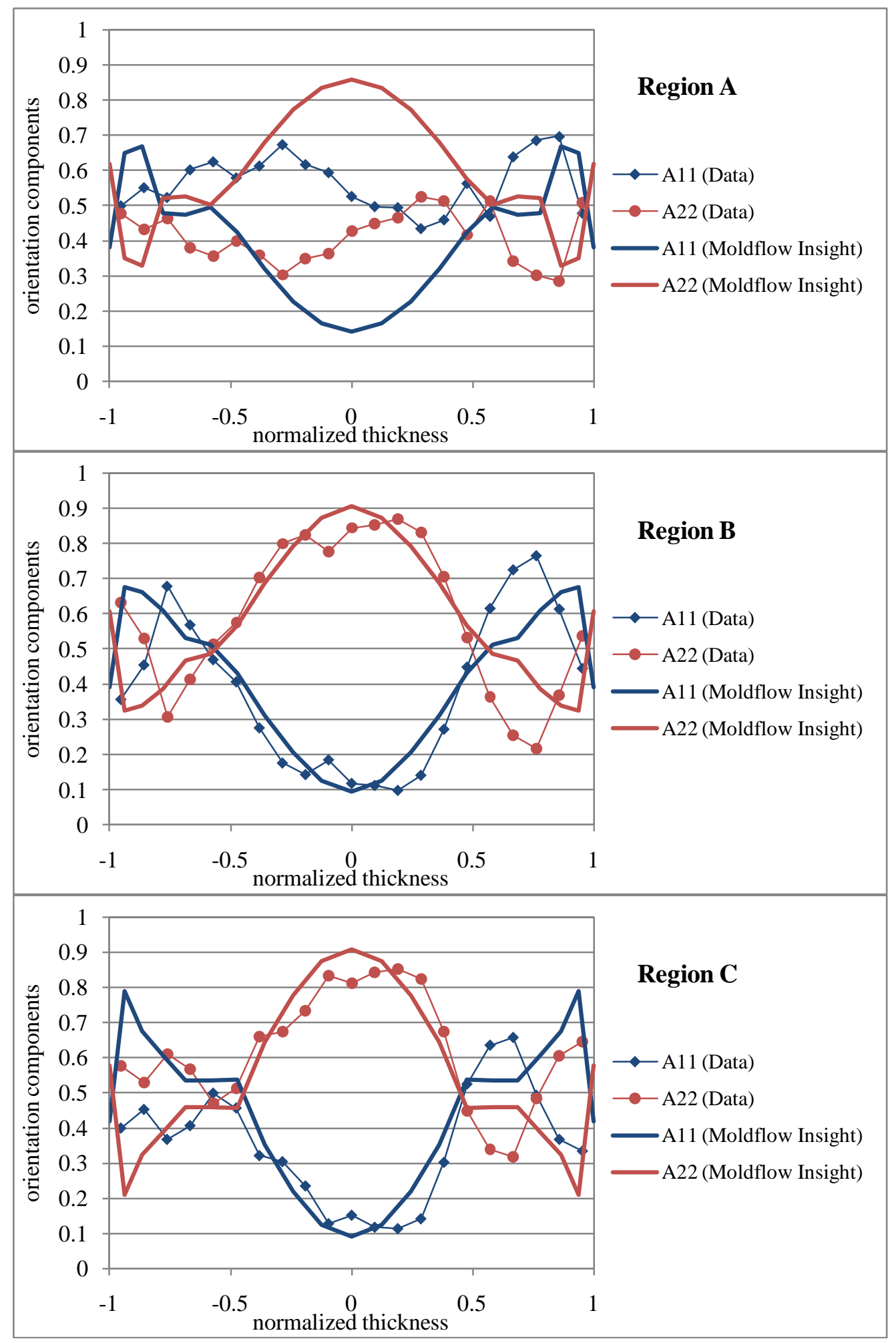

Figure 23 Comparison of fiber orientation data with the prediction by the ARD-RSC model with $\kappa=1 / 30$ for the AS3D disk (slow fill) using a mid-plane mesh. 


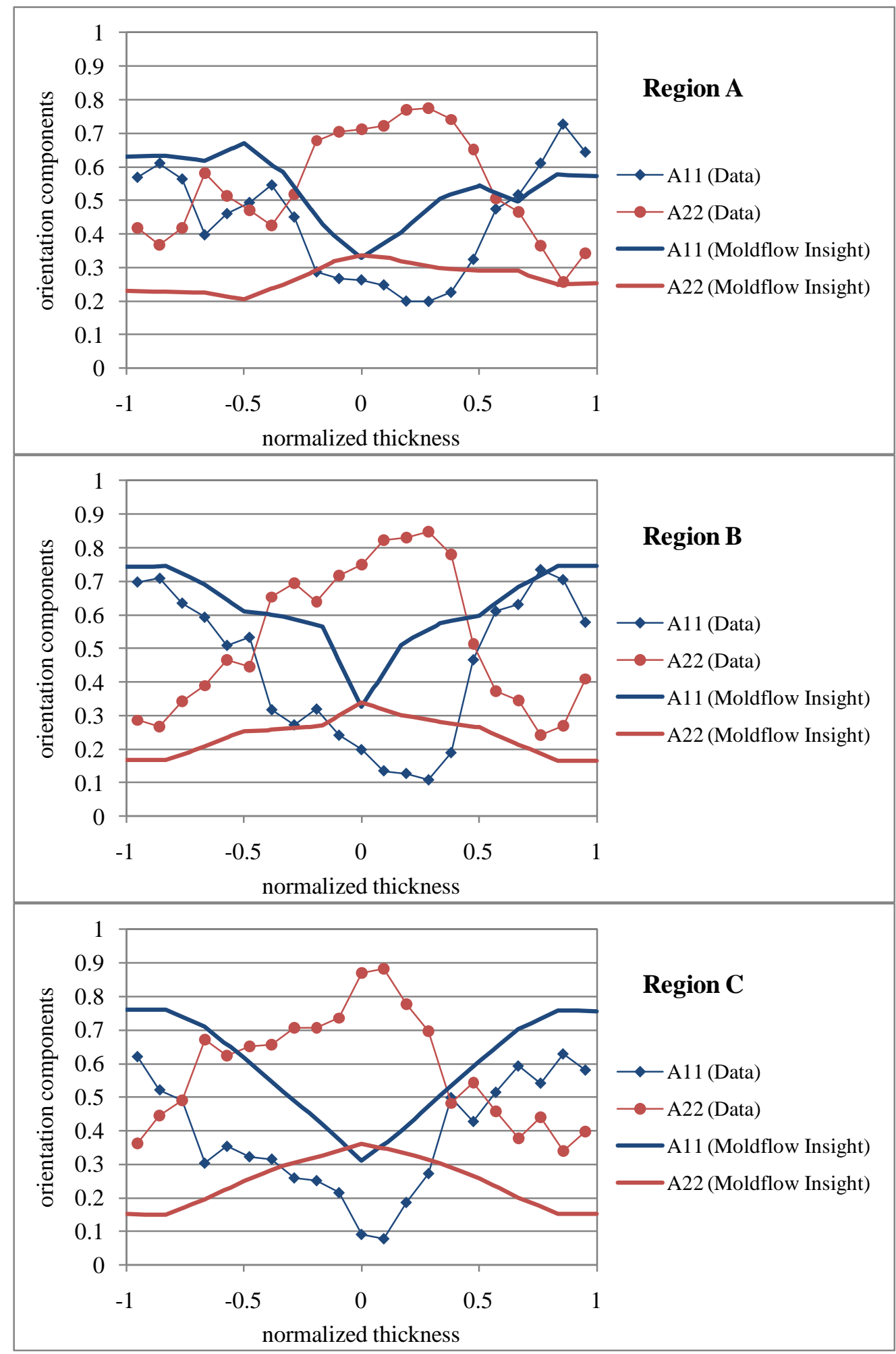

Figure 24 Comparison of fiber orientation data with the prediction by the ARD-RSC model with $\kappa=1 / 30$ for the AF3I plaque (fast fill) using a 3D mesh. 


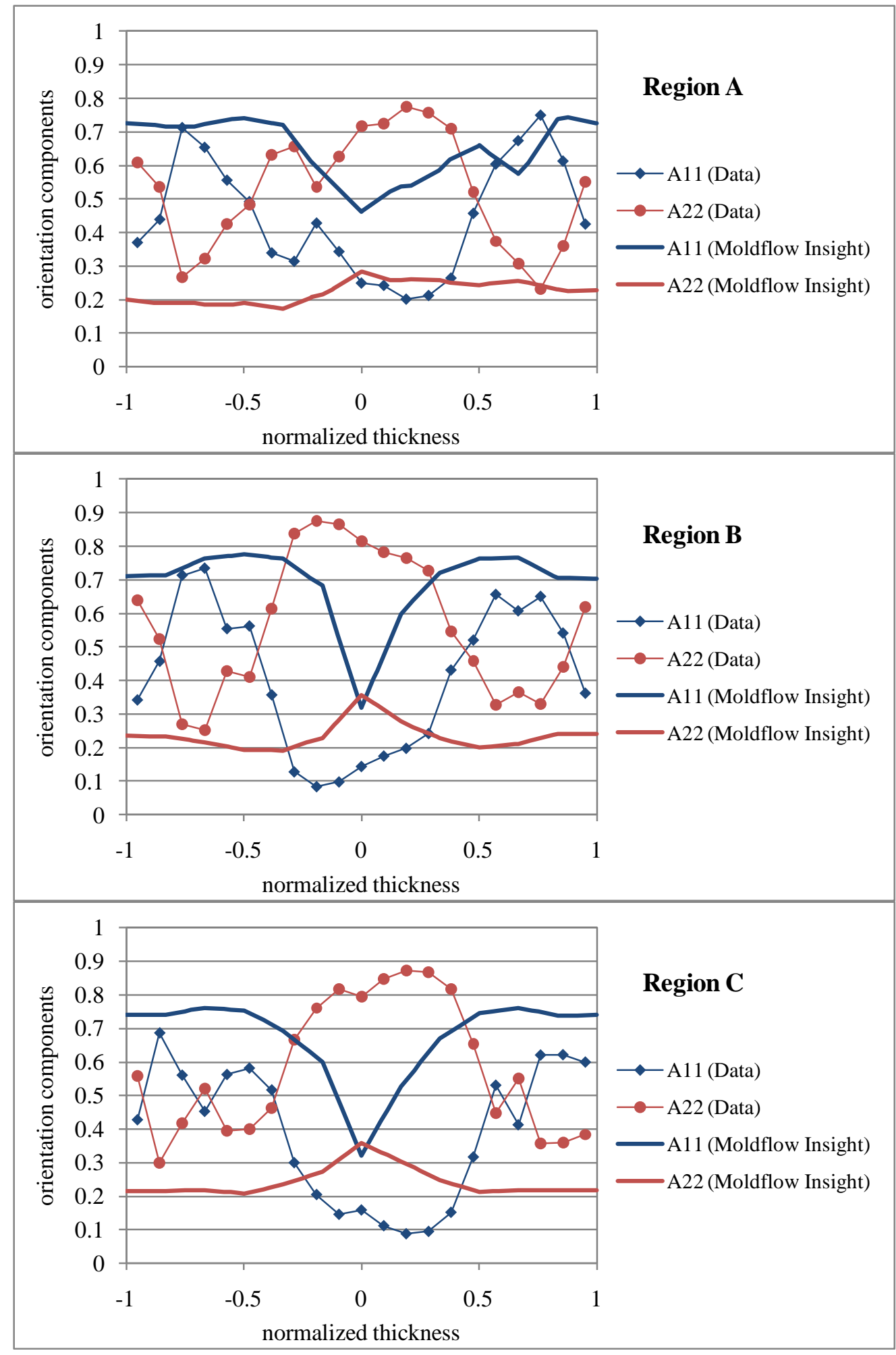

Figure 25 Comparison of fiber orientation data with the prediction by the ARD-RSC model with $\kappa=1 / 30$ for the AS3I plaque (slow fill) using a 3D mesh. 


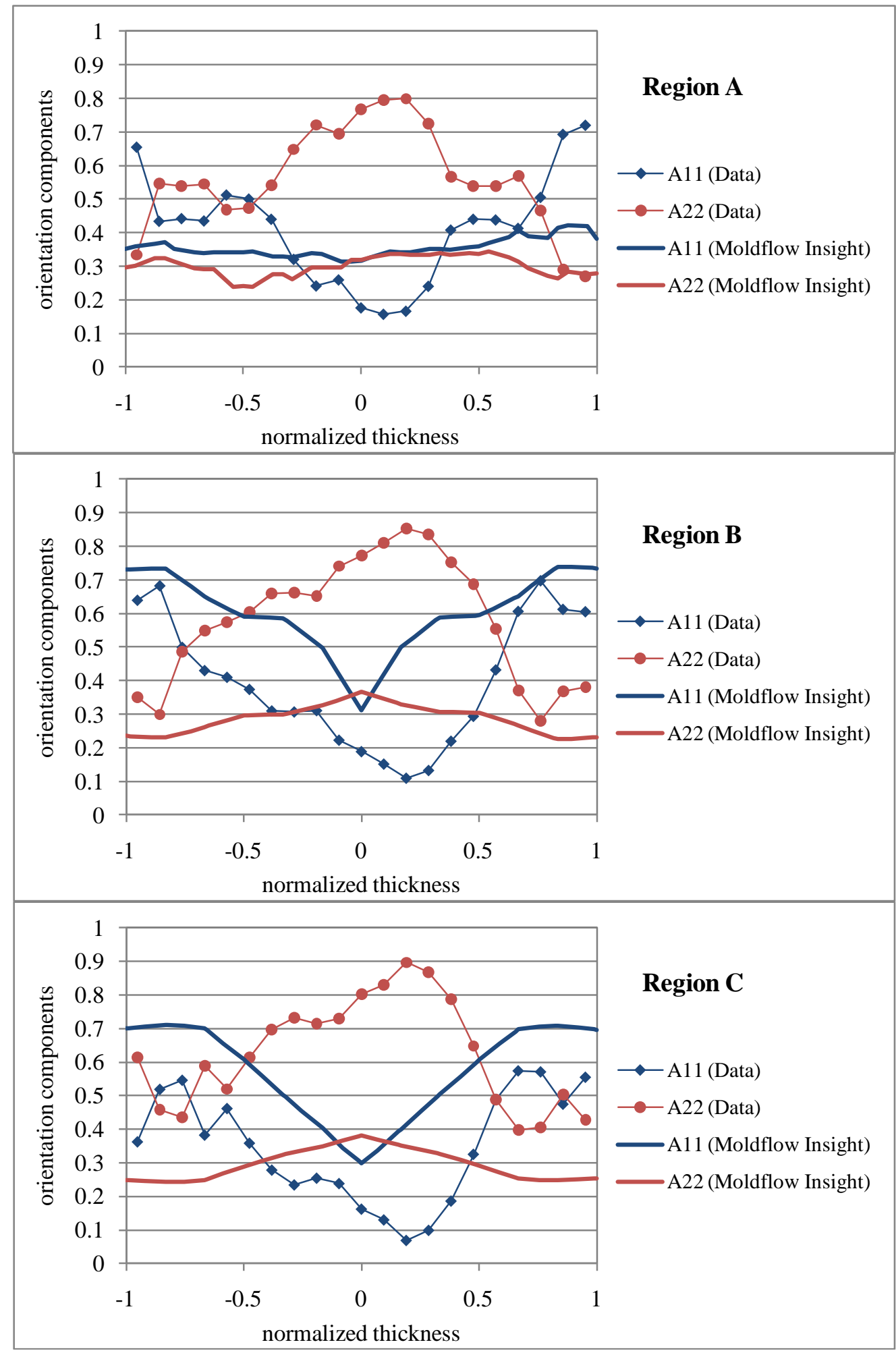

Figure 26 Comparison of fiber orientation data with the prediction by the ARD-RSC model with $\kappa=1 / 30$ for the AF3D disk (fast fill) using a 3D mesh. 


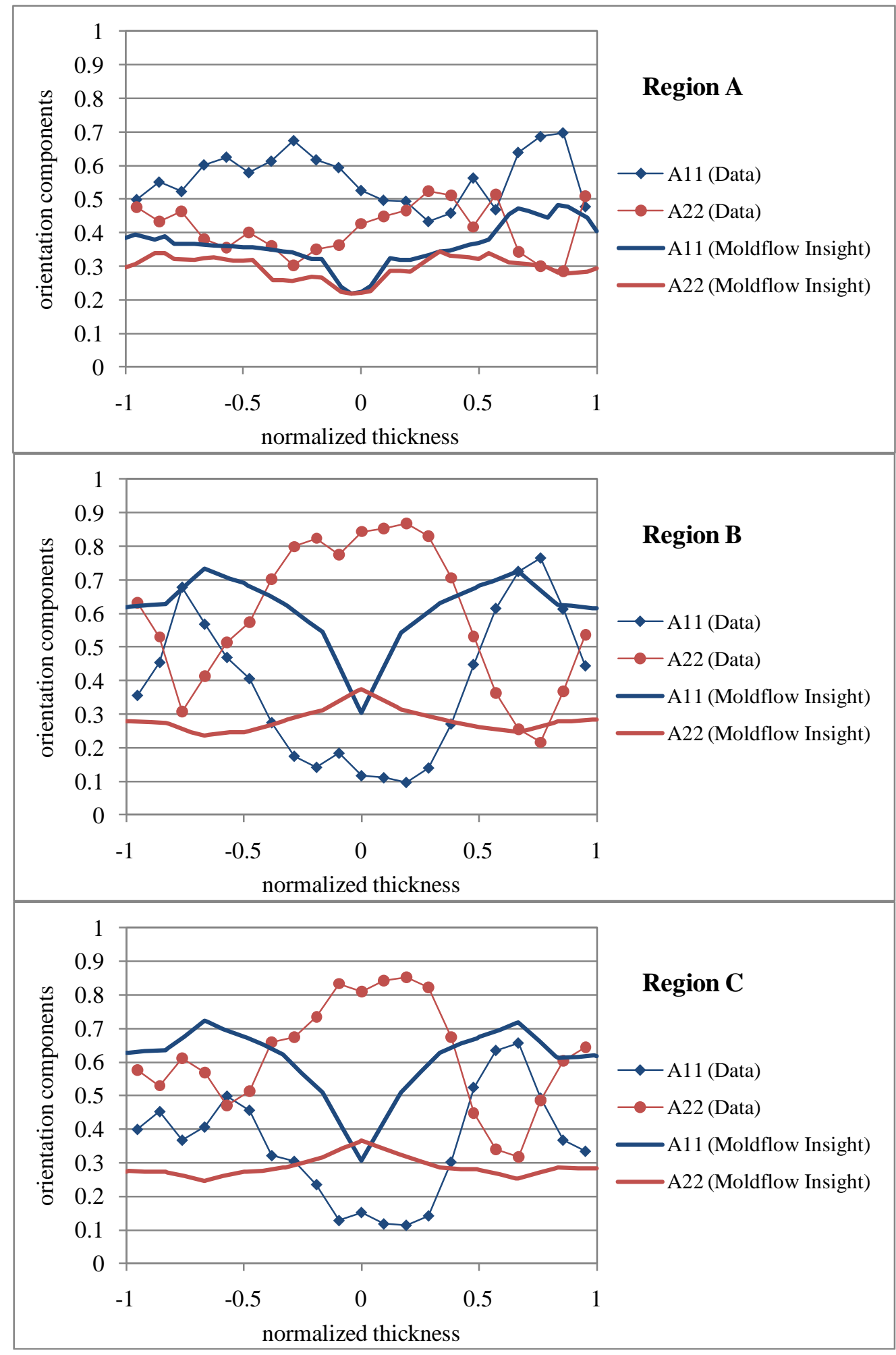

Figure 27 Comparison of fiber orientation data with the prediction by the ARD-RSC model with $\kappa=1 / 30$ for the AS3D disk (slow fill) using a 3D mesh. 


\subsection{Fiber Length Attrition Model Implementation}

The basic equation for solving the number distribution of fiber length segments is given by Phelps and Tucker [6-7], and it is written as

$$
\begin{aligned}
& \bar{N}_{i, j+1}=\bar{N}_{i, j}-\bar{P}_{i} \bar{N}_{i, j} \Delta t+\sum_{k \mid k \geq i}^{M} \bar{R}_{i k} \bar{N}_{k, j} \Delta t \\
& i=1,2, \ldots, M ;
\end{aligned}
$$

where $M$ is the number of length segments, and

$$
R_{i k}=N\left(l_{i}, \frac{l_{i}}{2}, S l_{i}\right)
$$

$S$ is dimensionless parameter that controls the shape of the Gaussian breakage profile, and $\bar{P}_{i}$ is given by Equation (9).

\section{Influence of the Number of Length Segments}

It can be noted that the number of length segments, $M$, is less influential comparing the curves of $M=10$ with the curves of $M=100$ in the average length curve plot shown in Figure 28.

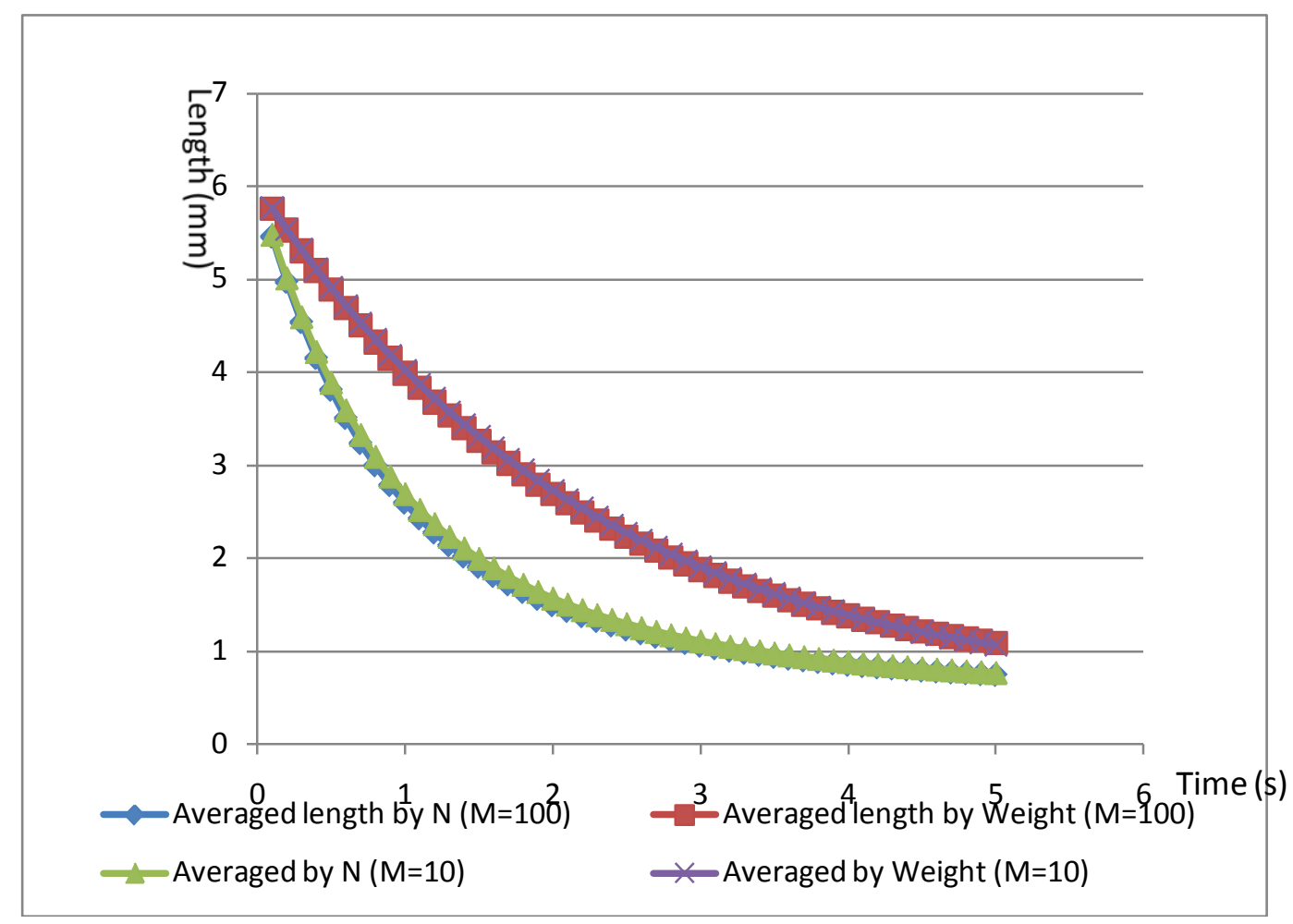

Figure 28 Average length curves with $M=10$ and $M=100$.

For the model implementation, it can be seen that the number of length segments in array $N$ can be a big cost in terms of memory allocation (Figure 29). For a mid-plane/dual domain solver implementation, it requires two arrays of $N$ at each laminate of each element, that is, if the 
number of length segments is 10, it requires 400 times of element count to store the variation of length segments for the fiber length breakage calculation, and 20 times of tetra element count for the 3D fiber breakage calculation. Currently, a variable is set for comparison. The default is 10 segments for a midplane/dual domain mesh, and 20 for a 3D mesh. The implementation has been carried out in such a way that it can handle certain case studies, but it is potentially a problem if the mesh for the analysis is very fine.

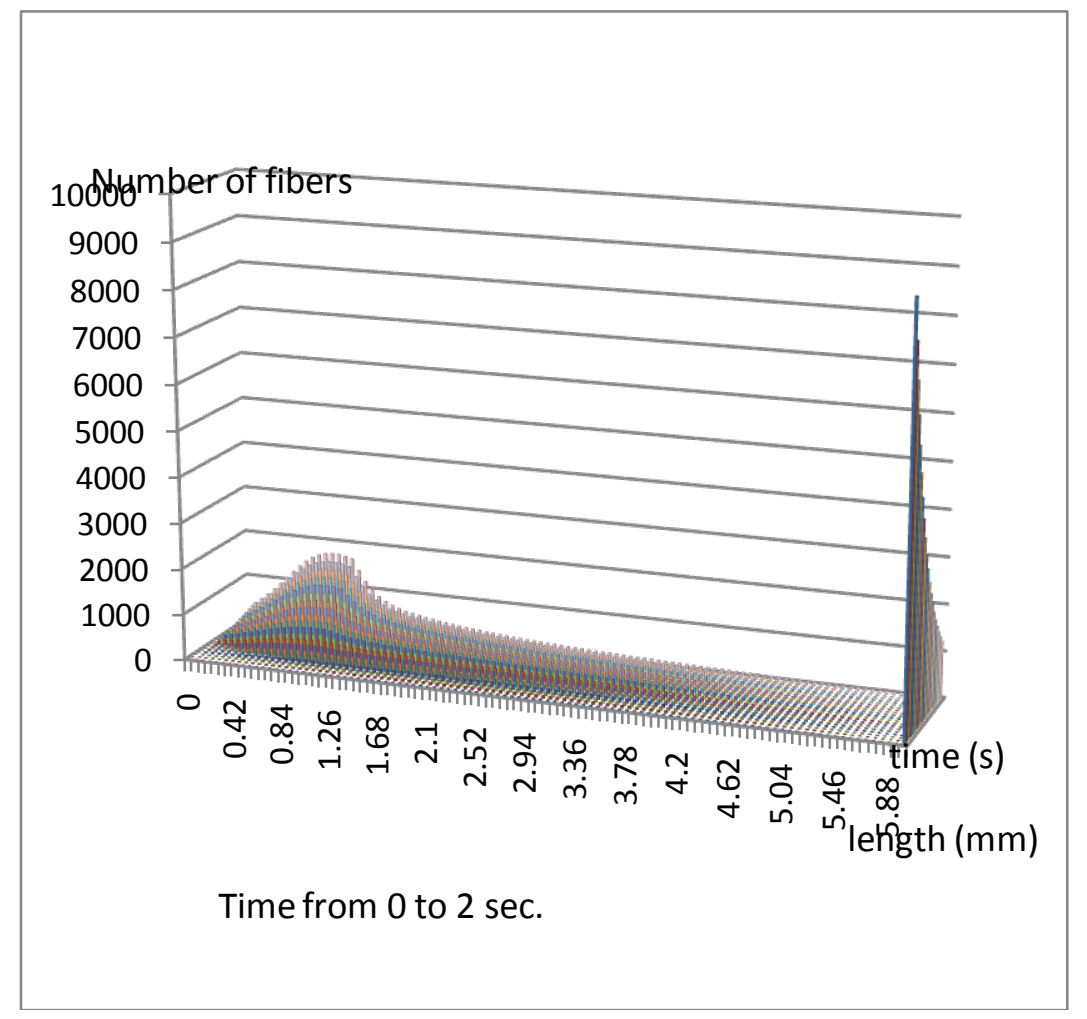

Figure 29 Fiber length distribution with time and segments

\section{$\underline{\text { Transport Term }}$}

A convection term is added in Equation (11) due to the reason that the broken fibers are transported downstream, and for a numerical scheme that uses the Eulerian approach, it is essential to have this term. Equation (11) can also be written in the following functional form:

$$
\frac{\partial f(x, t)}{\partial t}=-P(x) f(x, t)+\int_{x}^{L} R(x, y) f(y, t) d y
$$

Adding a convection term, Equation (13) is completed as:

$$
\frac{\partial f(x, t)}{\partial t}+\vec{v} \cdot \nabla f(x, t)=-P(x) f(x, t)+\int_{x}^{L} R(x, y) f(y, t) d y
$$

where $x$ is the length variable, $L$ the initial length. Equation (14) has been implemented in Autodesk Moldflow Insight instead of the original Equation (11). 


\section{Time step for explicit scheme}

The time step cannot be any value in the current explicit numerical scheme used in fiber length calculations. Of course it has to be positive, but whether it can be the same as the time step value that the flow solver uses can be analyzed as follows. By examining Equation (11), again it can be seen that the number of different lengths cannot be a negative value, though it can well drop down to zero, thus

$$
\begin{aligned}
& \bar{N}_{i, j}-\bar{P}_{i} \bar{N}_{i, j} \Delta t+\sum_{k \mid k \geq i}^{M} \bar{R}_{i k} \bar{N}_{k, j} \Delta t \geq 0 \\
& i=1,2, \ldots, M ;
\end{aligned}
$$

This indicates that the time step has to satisfy the following condition:

$$
\Delta t \leq \min \left\{\frac{N_{i, j}}{\bar{P}_{i} N_{i, j}-\sum_{k \mid k \geq i}^{M} \bar{R}_{i k} \bar{N}_{k, j}}\right\}, N_{i, j}>0
$$

This time step for time marching has been implemented to render the calculation stable.

\section{Fountain flow effect}

It is clear that the fountain effect in the flow front affects the fiber length results at and near the wall. Without the fountain effect, the fiber length distribution at wall nodes is not calculated because the polymer is "frozen" at these nodes. The fountain effect has been implemented to account for the skin frozen-in breakage. A good convection scheme can be seen from the fiber length result that the downstream area should not be longer than its upstream, except in the very skin and surface area that the fountain flow effect manifests (Figure 30). However, some long fibers can still be observed near the end of cavity, in the ribs, and in the weld surface area as shown in Figures 31 and 32.

By observing the convection and fountain effects, some long fibers are carried over downstream even though they are going through a narrow gate or runner, those in the center core layers can be pushed down quickly enough without breaking down, and remain quite long in the core layers or get frozen as soon as they are turned onto the skin area. Therefore it is important to have both the convection term and fountain effect implemented. 


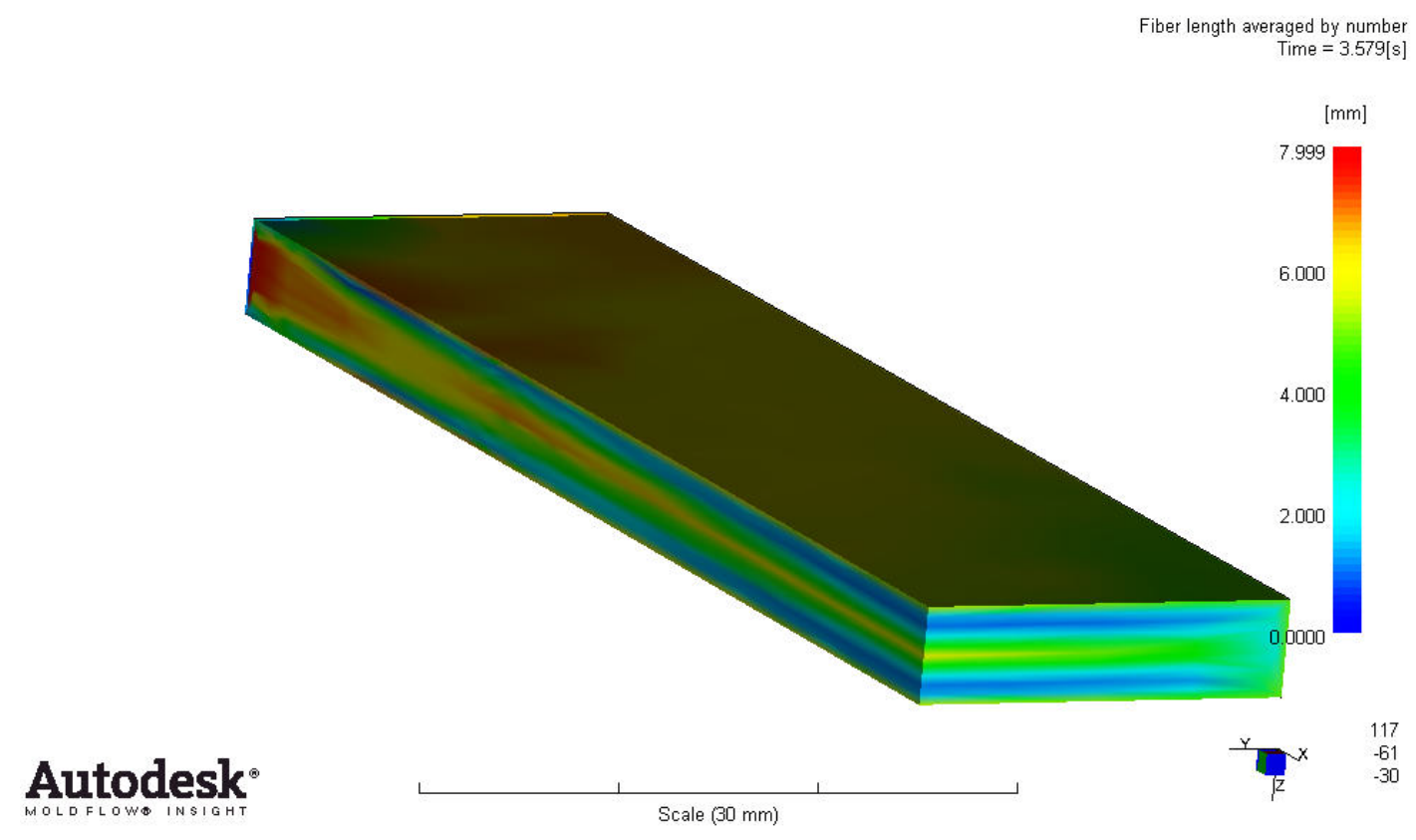

Figure 30 Fiber breakage results in two cross sectional views.

Fiber length averaged by number
Time $=3.579[\mathrm{~s}]$

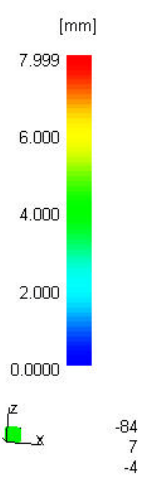

\section{Autodesk}

Scale $(20 \mathrm{~mm})$

Figure 31 Fiber breakage result at the end of cavity.

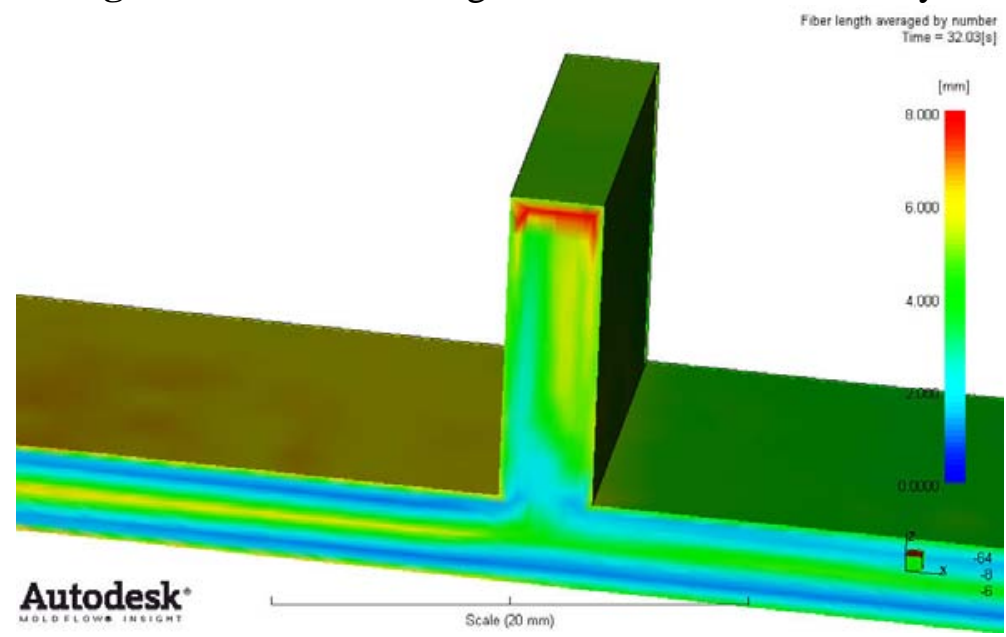

Figure 32 Fiber breakage results in a rib and at the top of the rib. 


\subsection{Dialogue Box Designs for FOD and FLD}

The material dialog box for long fiber filled materials has been added as a unique section, under "Filler Properties" tag, for viewing and editing initial fiber length, measurement method, and year of measurement as shown in Figure 33. The initial fiber length is needed for the fiber length attrition model, and for automatically determining what fiber orientation model to be used. The fiber length threshold for using long fiber models, according to Prof. Tucker's suggestion in a private communication, is hard-coded as $1 \mathrm{~mm}$. Thus if the initial fiber length is not shorter than $1 \mathrm{~mm}$, the fiber length attrition model will be triggered and if the default "autodetermine" remains unchanged in the fiber dialog box, the ARD fiber orientation model will be used.

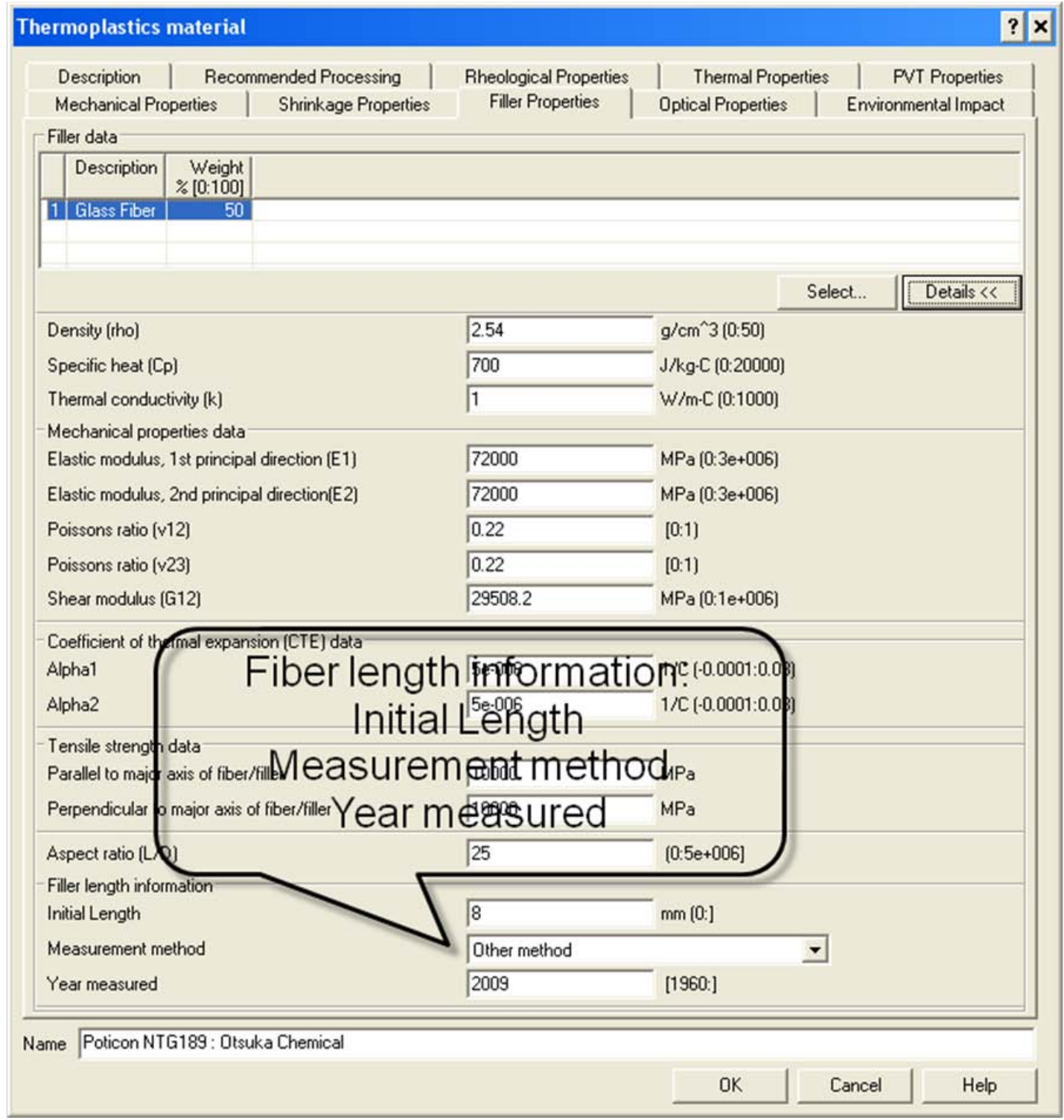

Figure 33 Dialog box for fiber length information data. 
It can be seen from the selection list of "Fiber Solver Parameters" dialog box (Figure 34) that the default option is the "Auto-determined model based on fiber length". The other options require user to input at least a parameter, such as the interaction coefficient $\mathrm{C}_{\mathrm{I}}$, or the reduced strain closure parameter. "Dz" is the thickness moment parameter that Autodesk Moldflow Insight modified the Folgar-Tucker model for mid-plane/dual domain mesh uses.

Fiber length attrition model parameters can be seen in the following dialog box as in the added section shown in Figure 35. There are three parameters: drag coefficient Dg ( $\varsigma$ in $F_{i}$ ), strain rate coefficient $C_{\mathrm{b}}$, and the distribution profile controller $S$ of Equation (12). In the 3D version, the user-defined fiber length distribution at inlet is added as an option for research purpose, the default option for both the mid-plane/dual domain and 3D versions is to use the initial fiber length provided in the dialog box shown in Figure 33.

Inlet conditions are important for the new fiber orientation models such as the RSC and ARD-RSC models. Figure 36 shows where a user-defined fiber inlet condition can be set. The other two options are typically used by users who do not have the measured inlet condition.

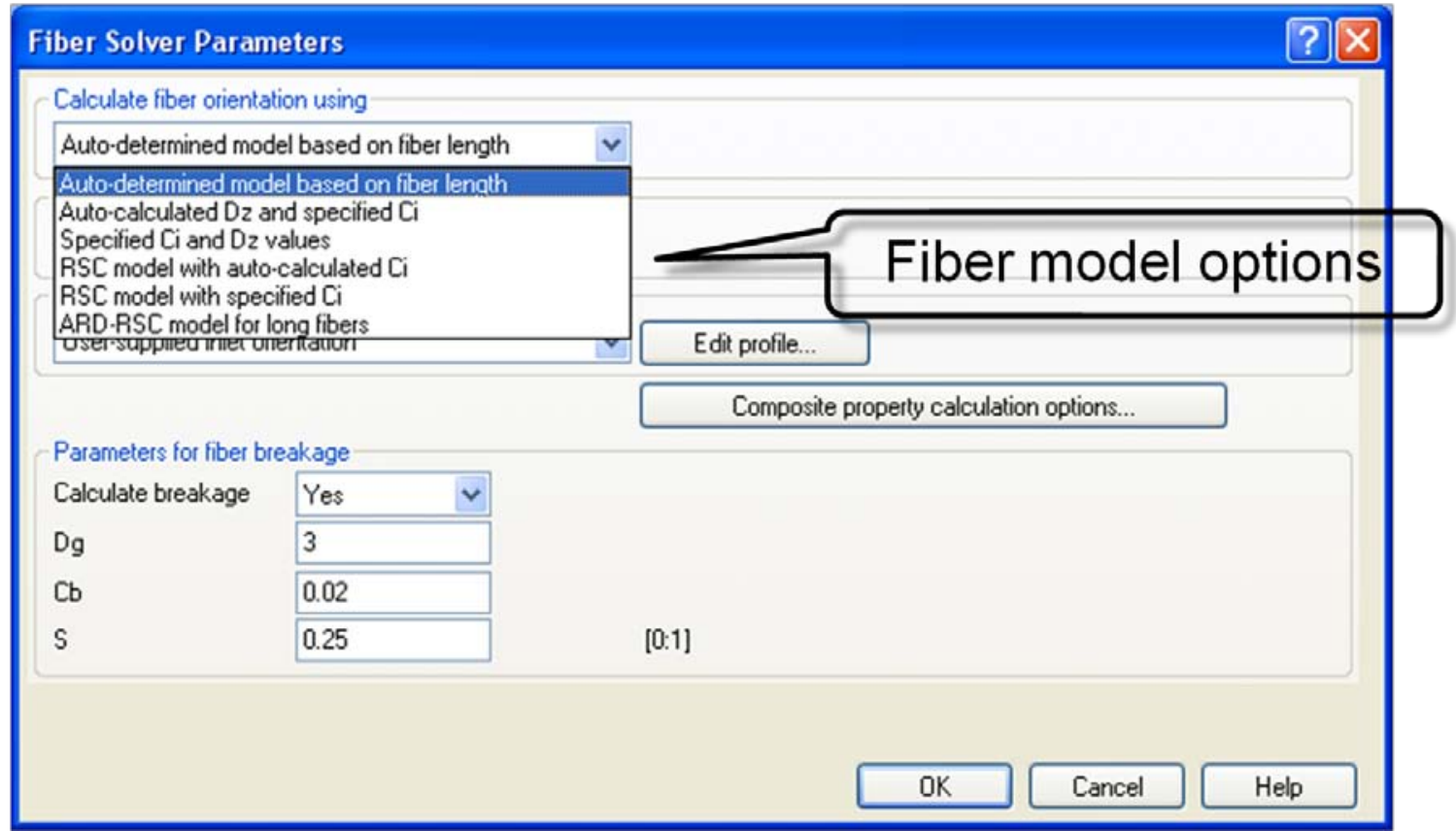

Figure 34 Dialog box for Fiber Solver Parameters. 


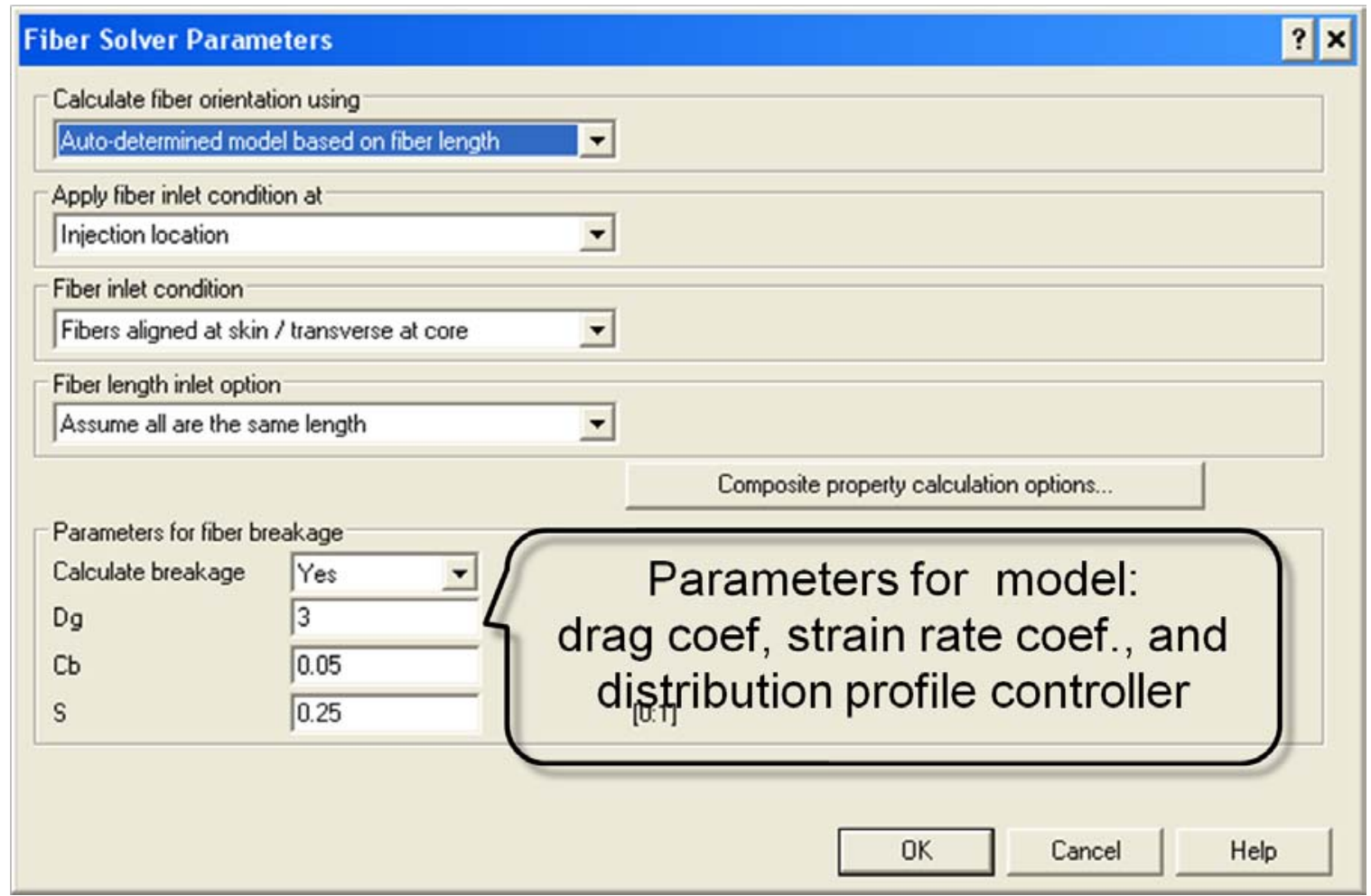

Figure 35 Fiber length attrition model parameters in Fiber Solver Parameters dialog box. 

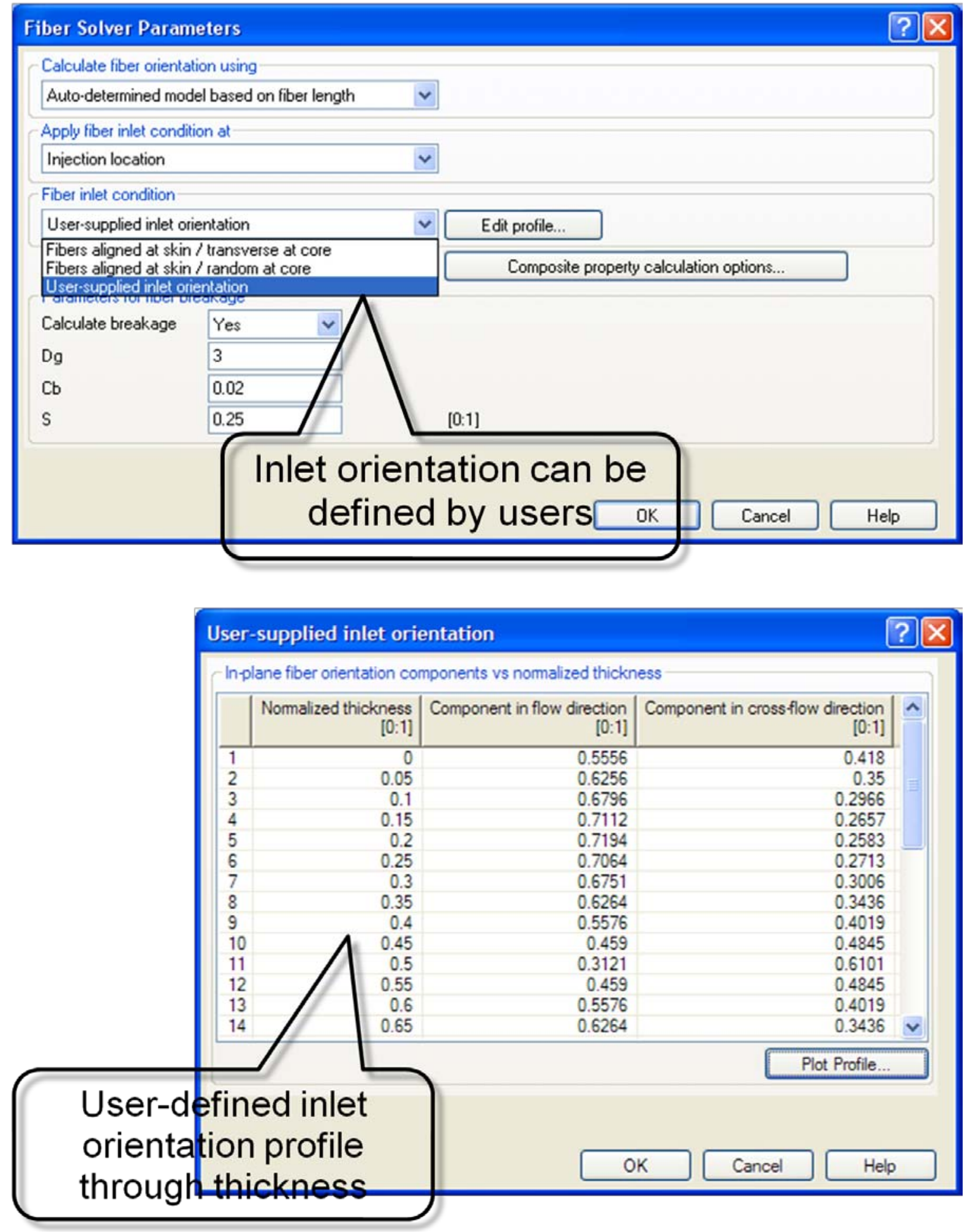

Figure 36 User-defined inlet condition. 
Reduced Strain Closure factor (RSC, the inverse value of SRF factor) and the $b_{\text {i }}$ parameters in ARD model can be introduced in the following dialog box (Figure 37).

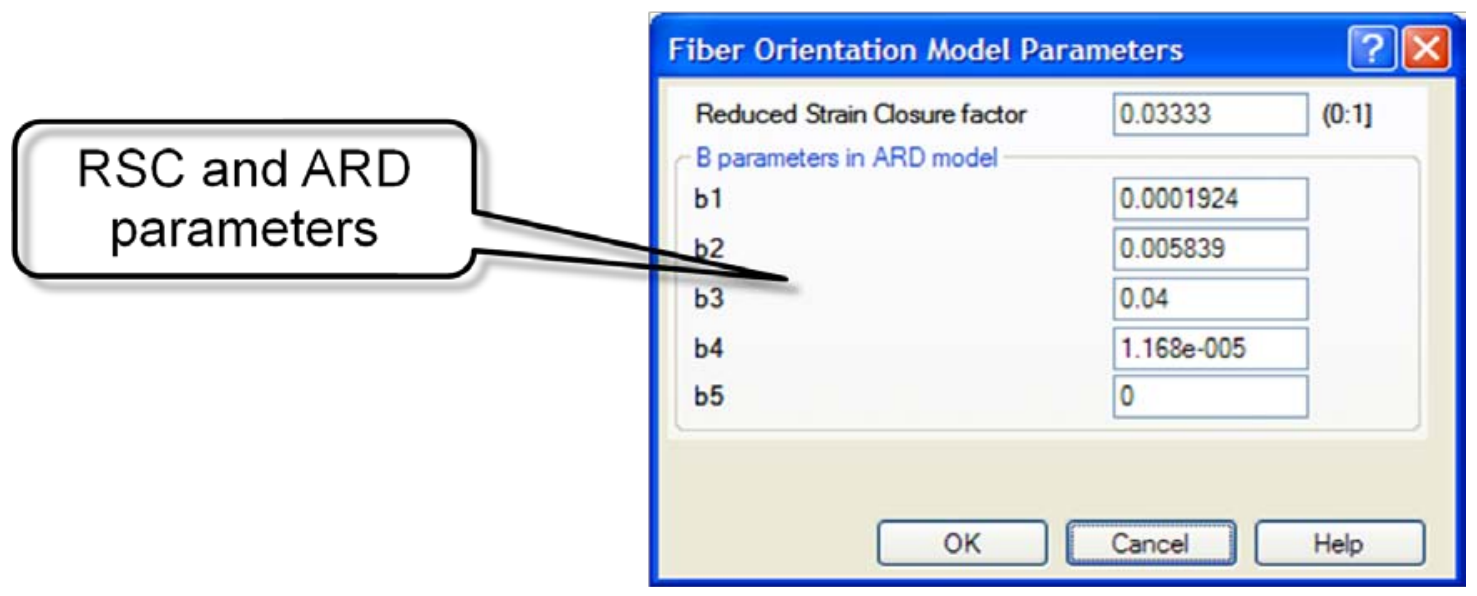

Figure 37 Dialog box for the RSC and ARD models’ parameters.

After the flow solution analysis is completed, typically fiber orientation tensor results are listed among the flow analysis result list if the material is a fiber-filled material. However, fiber length results are new and they are not in the default result list. To display the fiber length distribution results, one needs to go to "Results" to create a new plot, and select "Fiber length averaged by number" (or "Fiber length averaged by weight") from the complete list of all possible results, as shown in Figures 38 and 39.

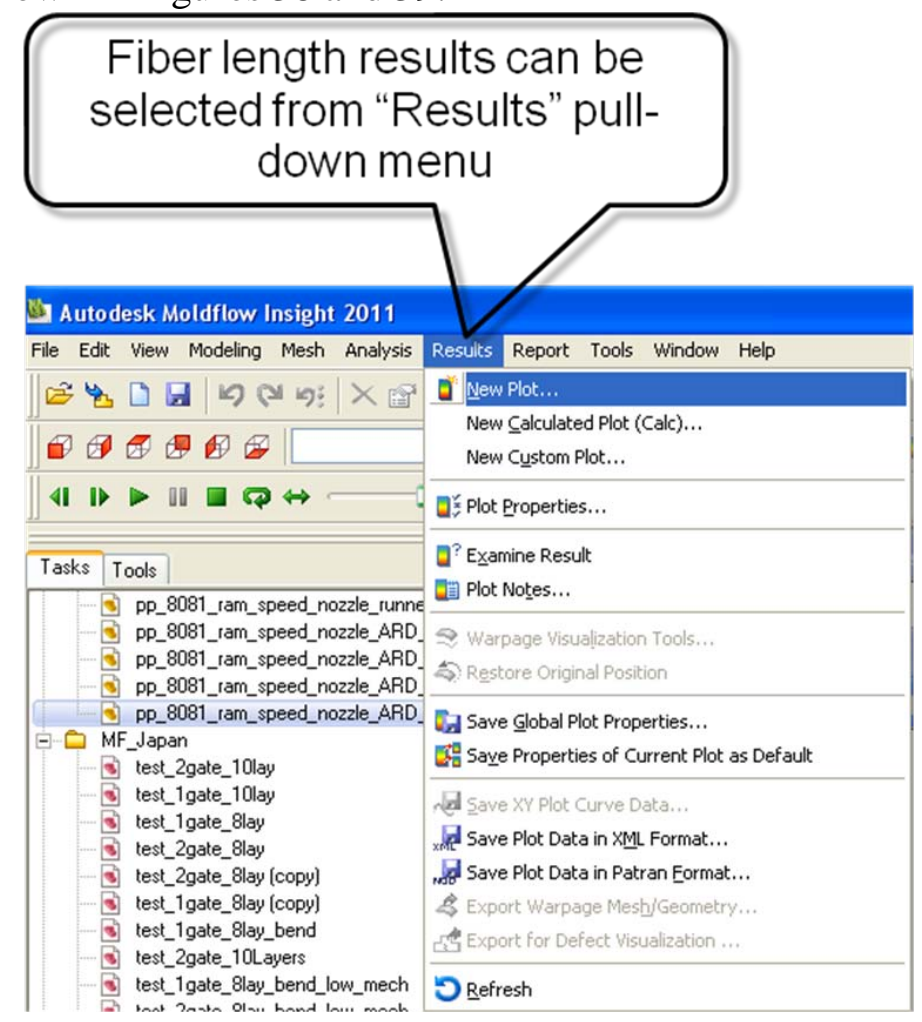

Figure 38 "New Plot” selection for for fiber length results from "Results”. 


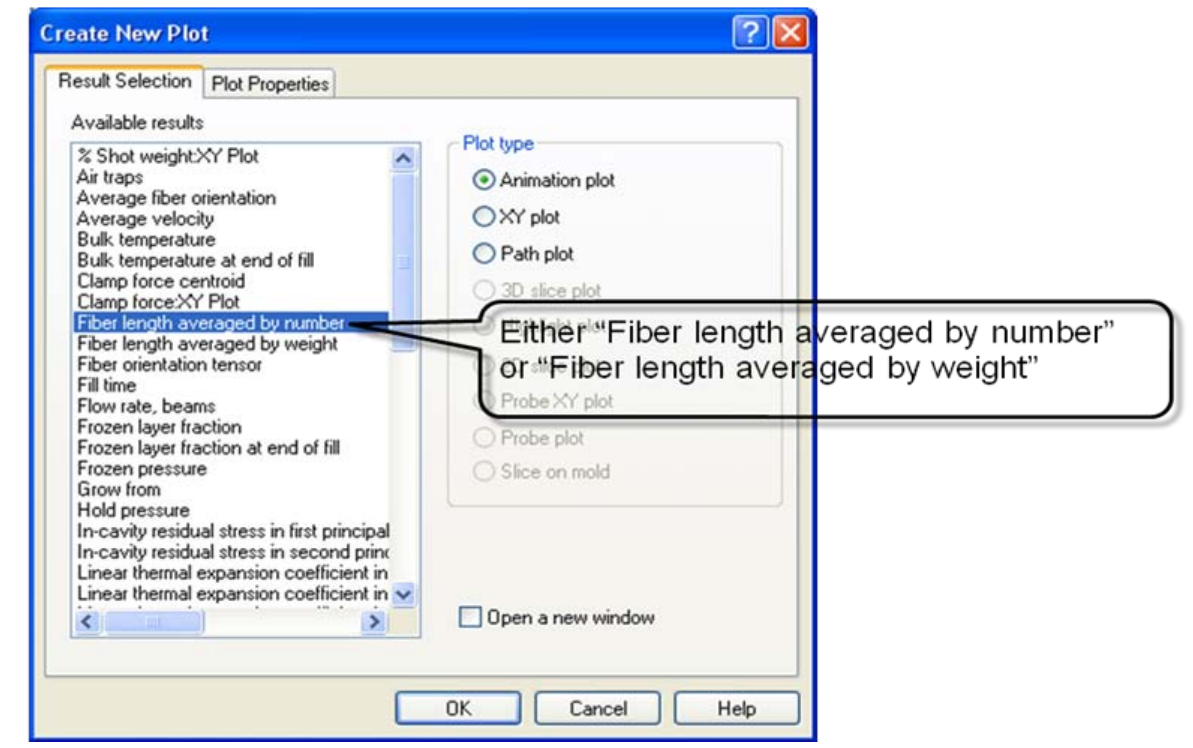

Figure 39 Fiber length distribution result selected from the complete result list.

\subsection{Conclusions}

Process models for fiber orientation and length distributions have been developed and implemented in the process modeling software packages (e.g. Autodesk Moldflow Insight) for injection-molded LFTs under the CRADA Nr. 260 between PNNL and Autodesk Inc. The fiber orientation model termed the ARD-RSC model uses the anisotropic rotary diffusion represented by a fiber-fiber interaction tensor allowing the fiber orientation in LFTs to be accurately predicted. The fiber orientation state in LFTs characterized by a large core layer which is rather oriented in the cross-flow direction while the shell layers are much thinner and where the fibers are more aligned with the flow direction. Numerical assessment has shown that neither the standard Folgar-Tucker model nor the RSC model by Wang et al. is capable to accurately capture all the components of the orientation tensor. The ARD-RSC model thus presents a great advancement in the state-of-the-art of modeling fiber orientation in injection-molded LFTs.

Due to the nature of the RSC and ARD-RSC models, the inlet condition strongly influences the fiber orientation prediction using these models in the middle of the part. Another important numerical aspect that affects the prediction is related to the accuracy of the velocity gradient calculation. Efforts have been made to address and solve these issues for the implementation of the fiber orientation models. As a result, fiber orientation predictions are in good agreements with the experimental results.

Next, a length breakage model for fibers in the mold cavity has been developed. This model takes into account the micromechanics of fiber breakage in a fiber suspension. It relates fiber orientation to the hydrodynamic loading of a fiber. This orientation-dependent model for fiber loading is subsequently related to a classical buckling criterion to predict fiber breakage. Finally, 
the fiber breakage model is implemented into an expression for the conservation of the total fiber length to derive a rate equation for fiber length distribution. Combined, this has then provided a fiber length attrition model in the mold cavity.

Special attentions have been paid to the convection term of the conservation equation for fiber length, fountain effect, and memory requirement for the implementation of the fiber breakage model. It should be mentioned that in the model mid-plane version of Autodesk Moldflow Insight, due to the limitation of the flow field calculation, the fountain flow effect is not sufficiently captured, and the memory requirement for large simulations could be an important issue to be solved in the future. It is found that fiber breakage mostly happens in the barrel and feed system of injection molding machines, thus the 3D implementation user defined inlet condition has been added to evaluate whether such a fiber length distribution at the inlet could influence the downstream fiber length distributions. The preliminary comparison of the prediction with the experimental fiber length data has shown that this fiber length attrition model can accurately capture the fiber length distribution along the flow length of the studied glassfiber/PP material. However, further assessment is needed, and it is necessary to further validate this model for other injection-molded materials.

\subsection{Acknowledgements}

The authors would like to thank the support of the US Department of Energy's Office of Vehicle Technologies - EERE, in particular, Dr. Joseph Carpenter Jr. and Dr. Carol Schutte.

\subsection{References}

[1] F. Folgar and C.L. Tucker III (1984). "Orientation Behavior of Fibers in Concentrated Suspensions,” Journal of Reinforced Plastic Composites, 3, 98-119.

[2] S.G. Advani and C.L. Tucker III (1987). "The Use of Tensors to Describe and Predict Fiber Orientation in Short-Fiber Composites,” Journal of Rheology, 31 (8), 751-784.

[3] J. Wang, J.F. O’Gara, and C.L. Tucker III (2008). “An Objective Model for Slow Orientation Kinetics in Concentrated Fiber Suspensions: Theory and Rheological Evidence," Journal of Rheology, 52(5):1179-1200.

[4] J.H. Phelps and C.L. Tucker III (2006). “Assessing Fiber Orientation Prediction Capability for Long-Fiber Thermoplastic Composites.” Technical Report submitted to PNNL, University of Illinois at Urbana-Champaign, IL 61801.

[5] J.H. Phelps and C.L. Tucker III (2009). “An Anisotropic Rotary Diffusion Model for Fiber Orientation in Short- and Long-Fiber Thermoplastics," Journal of the Non-Newtonian Fluid Mechanics, 156(3):165-176.

[6] J.H. Phelps (2009). "Processing-Microstructural Models for Short- and Long-Fiber Thermoplastic Composites,” PhD Thesis, University of Illinois at Urbana-Champaign, Urbana, IL 61801. 
[7] J.H. Phelps and C.L. Tucker III (2009). "Processing-Microstructure Modeling for Long-Fiber Thermoplastics," Technical Report submitted to PNNL, University of Illinois at UrbanaChampaign, IL 61801.

[8] J.S. Cintra Jr. and C.L. Tucker III (1995). "Orthotropic Closure Approximations for FlowInduced Fiber orientation, Journal of Rheology, 39: 1095-1122.

[9] E.D. Wetzel (1999). Modeling Flow-Induced Microstructure in Inhomogeneous LiquidLiquid Mixtures, PhD thesis, University of Illinois at Urbana-Champaign, Urbana, IL 61801.

[10] R.S. Bay (1991). "Fiber Orientation in Injection Molded Composites: A Comparison of Theory and Experiment,” PhD Thesis, University of Illinois at Urbana-Champaign, Urbana, IL 61801.

[11] R. S. Bay and C. L. Tucker III (1992). "Fiber Orientation in Simple Injection Moldings. Part I: Theory and Numerical Methods,” Polymer Composites, 13:317-331.

[12] Moldflow Material Testing Report MAT2586 - PP-40G prepared for the Pacific Northwest National Laboratory by Moldflow Plastics Labs, Kilsyth, Victoria, Australia.

[13] P.J. Hine, N. Davidson, R. A. Duckett, A. R. Clarke, and I. M. Ward (1996). "Hydrostatically Extruded Glass-Fiber-Reinforced Polyoxymethylene - I: The development of Fiber and Matrix Orientation,” Polymer Composites, 17:720-729.

[14] N. Phan-Thien, X.-J. Fan, R.I. Tanner, and R. Zheng (2002). "Folgar-Tucker Constant for a Fibre Suspension in a Newtonian Fluid,” Journal of Non-Newtonian Fluid Mechanics, 103:251-260.

[15] S.M. Dinh and RC Armstrong (1984). "A Rheological Equation of State for SemiConcentrated Fiber Suspensions,” Journal of Rheology, 28(3):207-227.

[16] J. Wang (2007). “Improved Fiber Orientation Predictions for Injection Molded Composites,” PhD Thesis, University of Illinois at Urbana-Champaign, Urbana, IL 61801. 